MATHEMATICS OF COMPUTATION

Volume 67, Number 221, January 1998, Pages 111-136

S 0025-5718(98)00901-6

\title{
FINITE ELEMENT ANALYSIS OF COMPRESSIBLE AND INCOMPRESSIBLE FLUID-SOLID SYSTEMS
}

\author{
ALFREDO BERMÚDEZ, RICARDO DURÁN, AND RODOLFO RODRÍGUEZ
}

\begin{abstract}
This paper deals with a finite element method to solve interior fluid-structure vibration problems valid for compressible and incompressible fluids. It is based on a displacement formulation for both the fluid and the solid. The pressure of the fluid is also used as a variable for the theoretical analysis yielding a well posed mixed linear eigenvalue problem. Lowest order triangular Raviart-Thomas elements are used for the fluid and classical piecewise linear elements for the solid. Transmission conditions at the fluid-solid interface are taken into account in a weak sense yielding a nonconforming discretization. The method does not present spurious or circulation modes for nonzero frequencies. Convergence is proved and error estimates independent of the acoustic speed are given. For incompressible fluids, a convenient equivalent stream function formulation and a post-process to compute the pressure are introduced.
\end{abstract}

\section{INTRODUCTION}

Increasing attention has recently been paid to problems involving fluid-structure interactions. For a survey of current results see [12] and references therein. In this paper, we are concerned with the interaction between a fluid, either compressible or incompressible, contained in an elastic structure (e.g., the internal elastoacoustics or hydroelasticity problems).

We consider as a model problem a 2D elastic vessel completely filled by a fluid. Displacement variables are used for both the fluid and the solid; however, to provide a theoretical analysis, also the pressure in the fluid is used as a variable.

Under the usual assumptions leading to linear problems, the evolution of both the fluid and the structure is governed by second order in time linear equations. Their solution can be written in terms of the vibration modes of the coupled system which are eigenfunctions of a linear eigenvalue problem.

When a displacement formulation is discretized, spurious modes use to appear; this is the case, for instance, if continuous piecewise linear finite elements are used for both the fluid and the solid (see [11] and [3]). Such spurious modes are approximations of pure rotational motions of the fluid which can be seen as zero frequency eigenmodes of the continuous problem. These rotational eigenmodes are not relevant from a physical viewpoint. However, when the discrete problem does not have zero as an eigenfrequency with a corresponding eigenspace approximating this set

Received by the editor March 6, 1995 and, in revised form, May 22, 1996.

1991 Mathematics Subject Classification. Primary 65N25, 65N30; Secondary 70J30, 73K70, $76 \mathrm{Q} 05$. 
of rotational motions, spurious eigenmodes arise with nonzero frequencies placed among those of the relevant ones.

In [1] a finite element method which does not present spurious modes is introduced for the case of a compressible fluid. It consists in using piecewise linear elements for the solid and Raviart-Thomas elements of lowest order for the fluid, the coupling of both being of nonconforming type. Such discretization yields a linear symmetric eigenvalue problem.

We show that this method can be adapted to deal with incompressible fluids too. In spite of the fact that incompressible fluids are the limit case of compressible ones when the acoustic speed goes to infinity, the proofs in [1] have to be modified since the constants in the estimates therein blow up with the acoustic speed.

We present an alternative approach covering both the compressible and the incompressible cases. We prove the convergence of our method and error estimates not depending on the acoustic speed are given. We also prove that spurious modes do not arise when sufficiently refined meshes are used. We analyze the asymptotic behavior of the eigenfrequencies in the compressible case as the acoustic speed goes to infinity. Finally, we discuss implementation issues in the incompressible case. Numerical experiments showing the effectiveness of the method are reported in [3] for compressible fluids and in [2] for incompressible ones.

\section{THE MODEL PROBLEM}

We consider the problem of determining the vibration modes of a linear elastic structure containing an ideal (inviscid) barotropic fluid; the fluid can be compressible or incompressible. Our model problem consists of a $2 \mathrm{D}$ polygonal vessel completely filled with the fluid as that in Fig. 1.

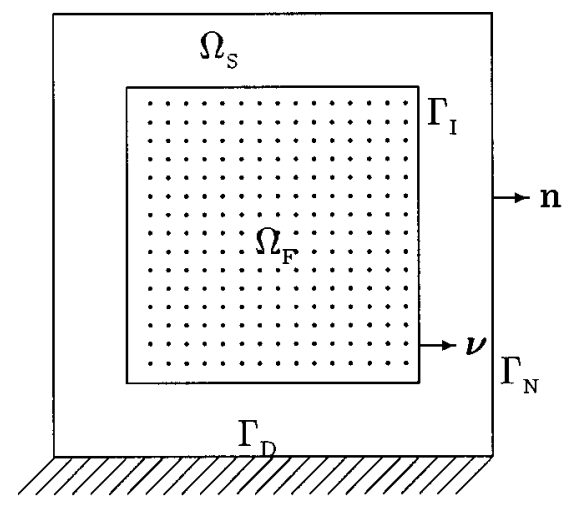

FiguRE 1. Fluid and solid domains.

Let $\Omega_{\mathrm{F}}$ and $\Omega_{\mathrm{S}}$ be the domains occupied by the fluid and the solid respectively as in Fig. 1. We assume $\Omega_{\mathrm{F}}$ to be simply connected but not necessarily convex. $\Gamma_{\mathrm{I}}$ denotes the interface between the solid and the fluid and $\boldsymbol{\nu}$ its unit normal vector pointing outwards $\Omega_{\mathrm{F}}$. We denote by $\Gamma_{j}, j=1, \ldots, J$, the edges of the polygonal interface $\Gamma_{\mathrm{I}}$; therefore, we have $\Gamma_{\mathrm{I}}=\bigcup_{j=1}^{J} \Gamma_{j}$. The exterior boundary of the solid is the union of $\Gamma_{\mathrm{D}} \neq \emptyset$ and $\Gamma_{\mathrm{N}}$ : the structure is fixed along $\Gamma_{\mathrm{D}}$ and free of stress along $\Gamma_{\mathrm{N}} ; \mathbf{n}$ denotes the unit outward normal vector along $\Gamma_{\mathrm{N}}$. 
Throughout this paper we use the standard notation for Sobolev spaces, norms and seminorms. We also denote $H\left(\operatorname{div}, \Omega_{\mathrm{F}}\right):=\left\{\mathbf{u} \in\left[L^{2}\left(\Omega_{\mathrm{F}}\right)\right]^{2}: \operatorname{div} \mathbf{u} \in L^{2}\left(\Omega_{\mathrm{F}}\right)\right\}$ and $\left.\|\mathbf{u}\|_{H\left(\operatorname{div}, \Omega_{\mathrm{F}}\right)}^{2}:=\|\mathbf{u}\|_{\left[L^{2}\left(\Omega_{\mathrm{F}}\right)\right.}^{2}\right]^{2}+\|\operatorname{div} \mathbf{u}\|_{L^{2}\left(\Omega_{\mathrm{F}}\right)}^{2}$. We denote by $\mathbf{C}$ a generic constant not necessarily the same at each occurrence.

We use the following notations for the physical magnitudes; in the fluid:

$\mathbf{u}$ : the displacement vector,

$p$ : the pressure,

$\rho_{\mathrm{F}}$ : the density,

$c$ : the acoustic speed $\left(c_{0} \leq c \leq \infty\right.$, with $c=\infty$ for an incompressible fluid) and in the solid:

$\mathbf{v}$ : the displacement vector,

$\rho_{\mathrm{S}}$ : the density,

$\lambda_{\mathrm{S}}$ and $\mu_{\mathrm{S}}$ : the Lamé coefficients,

$\varepsilon(\mathbf{v})$ : the strain tensor defined by $\varepsilon_{i j}(\mathbf{v}):=\frac{1}{2}\left(\frac{\partial v_{i}}{\partial x_{j}}+\frac{\partial v_{j}}{\partial x_{i}}\right)$,

$\boldsymbol{\sigma}(\mathbf{v})$ : the stress tensor which is related to the strain tensor by Hooke's law:

$$
\sigma_{i j}(\mathbf{v}):=\lambda_{\mathrm{s}} \sum_{k=1}^{2} \varepsilon_{k k}(\mathbf{v}) \delta_{i j}+2 \mu_{\mathrm{s}} \varepsilon_{i j}(\mathbf{v}), \quad i, j=1,2,
$$

$\operatorname{div}[\boldsymbol{\sigma}(\mathbf{v})]=\left(\lambda_{\mathrm{S}}+\mu_{\mathrm{S}}\right) \nabla(\operatorname{div} \mathbf{v})+\mu_{\mathrm{S}} \Delta \mathbf{v}$ : the linear elasticity operator.

In the case of a compressible fluid, the classical elastoacoustics approximation for small amplitude motions yields the following eigenvalue problem for the vibration modes of the coupled system and their corresponding frequencies $\omega$ (see, for instance, [4]).

SP: Find $\omega \geq 0, \mathbf{u} \in H\left(\operatorname{div}, \Omega_{\mathrm{F}}\right), \mathbf{v} \in\left[H^{1}\left(\Omega_{\mathrm{S}}\right)\right]^{2}$ and $p \in H^{1}\left(\Omega_{\mathrm{F}}\right),(\mathbf{u}, \mathbf{v}, p) \neq$ $(\mathbf{0}, \mathbf{0}, 0)$, such that:

$$
\begin{aligned}
\nabla p-\omega^{2} \rho_{\mathrm{F}} \mathbf{u}=\mathbf{0}, & \text { in } \Omega_{\mathrm{F}}, \\
\frac{1}{\rho_{\mathrm{F}} c^{2}} p+\operatorname{div} \mathbf{u}=0, & \text { in } \Omega_{\mathrm{F}}, \\
\operatorname{div}[\boldsymbol{\sigma}(\mathbf{v})]+\omega^{2} \rho_{\mathrm{S}} \mathbf{v}=\mathbf{0}, & \text { in } \Omega_{\mathrm{S}}, \\
\boldsymbol{\sigma}(\mathbf{v}) \boldsymbol{\nu}+p \boldsymbol{\nu}=\mathbf{0}, & \text { on } \Gamma_{\mathrm{I}}, \\
\mathbf{u} \cdot \boldsymbol{\nu}-\mathbf{v} \cdot \boldsymbol{\nu}=0, & \text { on } \Gamma_{\mathrm{I}}, \\
\boldsymbol{\sigma}(\mathbf{v}) \mathbf{n}=\mathbf{0}, & \text { on } \Gamma_{\mathrm{N}}, \\
\mathbf{v}=\mathbf{0}, & \text { on } \Gamma_{\mathrm{D}} .
\end{aligned}
$$

The coupling between the fluid and the structure is taken into account by equations (2.4) and (2.5). The first one relates normal stresses of the solid on the interface with the pressure into the fluid. The second one means that fluid and solid are in contact at the interface. In spite of the fact that equations (2.3)-(2.6) of problem SP must be understood in the sense of distributions, since $p \in H^{1}\left(\Omega_{\mathrm{F}}\right)$ and $\mathbf{v} \in\left[H^{1}\left(\Omega_{\mathrm{S}}\right)\right]^{2}$, these interface conditions are valid in the $L^{2}\left(\Gamma_{\mathrm{I}}\right)$ sense.

The problem with an incompressible fluid can be thought of as the limit case of the previous one as $c$ goes to infinity. In this case (2.2) should be replaced by the simple condition $\operatorname{div} \mathbf{u}=0$. In order to deal with both cases in a same framework we consider $\frac{1}{\rho_{\mathrm{F}} c^{2}}=0$ for an incompressible fluid (i.e., $c=\infty$ ); so $(2.2)$ also makes 
sense in this case. All that follows in this paper is valid for $c=\infty$ as well as for finite values of $c$.

\section{VARIATIONAL FORMULATION}

In order to state a variational formulation of problem SP we introduce the functional spaces $Q:=L^{2}\left(\Omega_{\mathrm{F}}\right), \mathbf{H}:=\left[L^{2}\left(\Omega_{\mathrm{F}}\right)\right]^{2} \times\left[L^{2}\left(\Omega_{\mathrm{S}}\right)\right]^{2}, \mathbf{X}:=H\left(\operatorname{div}, \Omega_{\mathrm{F}}\right) \times$ $\left[H_{\Gamma_{\mathrm{D}}}^{1}\left(\Omega_{\mathrm{S}}\right)\right]^{2}$ (where $H_{\Gamma_{\mathrm{D}}}^{1}\left(\Omega_{\mathrm{S}}\right)$ is the subspace of functions in $H^{1}\left(\Omega_{\mathrm{S}}\right)$ vanishing on $\left.\Gamma_{\mathrm{D}}\right)$ and

$$
\mathbf{V}:=\left\{(\mathbf{u}, \mathbf{v}) \in \mathbf{X}: \mathbf{u} \cdot \boldsymbol{\nu}=\mathbf{v} \cdot \boldsymbol{\nu}, \quad \text { on } \Gamma_{\mathrm{I}}\right\} .
$$

$\mathbf{V}$ is a closed subspace of $\mathbf{X}$. We denote by $\|\cdot\|$ the natural norm on $\mathbf{X}$, by $|\cdot|$ the $L^{2}$ norm on $\mathbf{H}$ or on $Q$ and by $(\cdot, \cdot)$ the corresponding inner products.

By using test functions $\boldsymbol{\phi}$ in $(2.1)$ and $\boldsymbol{\psi}$ in $(2.3)$ such that $(\boldsymbol{\phi}, \boldsymbol{\psi}) \in \mathbf{V}$, and $q \in Q$ in (2.2), the following mixed variational eigenvalue problem is obtained as a variational formulation of SP:

VP: Find $\lambda \in \mathbf{R},(\mathbf{u}, \mathbf{v}, p) \in \mathbf{V} \times Q,(\mathbf{u}, \mathbf{v}, p) \neq(\mathbf{0}, \mathbf{0}, 0)$, such that:

$$
\begin{aligned}
\int_{\Omega_{\mathrm{S}}} \boldsymbol{\sigma}(\mathbf{v}): \varepsilon(\boldsymbol{\psi})-\int_{\Omega_{\mathrm{F}}} p \operatorname{div} \boldsymbol{\phi}=\lambda\left(\int_{\Omega_{\mathrm{F}}} \rho_{\mathrm{F}} \mathbf{u} \cdot \boldsymbol{\phi}+\int_{\Omega_{\mathrm{S}}} \rho_{\mathrm{S}} \mathbf{v} \cdot \boldsymbol{\psi}\right), & \forall(\boldsymbol{\phi}, \boldsymbol{\psi}) \in \mathbf{V}, \\
-\int_{\Omega_{\mathrm{F}}} q \operatorname{div} \mathbf{u}-\frac{1}{\rho_{\mathrm{F}} c^{2}} \int_{\Omega_{\mathrm{F}}} p q=0, \quad \forall q \in Q, &
\end{aligned}
$$

where $\boldsymbol{\sigma}(\mathbf{v}): \boldsymbol{\varepsilon}(\boldsymbol{\psi}):=\sum_{i, j=1,2} \sigma_{i j}(\mathbf{v}) \varepsilon_{i j}(\boldsymbol{\psi})$ denotes the usual inner product. Let us remark once more that $1 /\left(\rho_{\mathrm{F}} c^{2}\right)=0$ for an incompressible fluid; then, in this case, (3.2) reduces to $-\int_{\Omega_{\mathrm{F}}} q$ div $\mathbf{u}=0$ for all $q \in Q$.

As we show below, VP is a mixed eigenvalue problem which does not satisfy one of Brezzi's classical conditions ensuring well-posedness. We introduce some further notation to analyze VP in this framework. We consider the following continuous bilinear forms:

$$
\begin{aligned}
a((\mathbf{u}, \mathbf{v}),(\boldsymbol{\phi}, \boldsymbol{\psi})) & :=\int_{\Omega_{\mathrm{S}}} \boldsymbol{\sigma}(\mathbf{v}): \varepsilon(\boldsymbol{\psi}), \quad(\mathbf{u}, \mathbf{v}),(\boldsymbol{\phi}, \boldsymbol{\psi}) \in \mathbf{X}, \\
b((\mathbf{u}, \mathbf{v}), q) & :=-\int_{\Omega_{\mathrm{F}}} q \operatorname{div} \mathbf{u}, \quad(\mathbf{u}, \mathbf{v}) \in \mathbf{X}, \quad q \in Q, \\
d((\mathbf{u}, \mathbf{v}),(\boldsymbol{\phi}, \boldsymbol{\psi})) & :=\int_{\Omega_{\mathrm{F}}} \rho_{\mathrm{F}} \mathbf{u} \cdot \boldsymbol{\phi}+\int_{\Omega_{\mathrm{S}}} \rho_{\mathrm{S}} \mathbf{v} \cdot \boldsymbol{\psi}, \quad(\mathbf{u}, \mathbf{v}),(\boldsymbol{\phi}, \boldsymbol{\psi}) \in \mathbf{H} .
\end{aligned}
$$

Form $a$ is symmetric and positive in the sense that $a((\mathbf{u}, \mathbf{v}),(\mathbf{u}, \mathbf{v})) \geq 0$ for all $(\mathbf{u}, \mathbf{v}) \in \mathbf{X}$. Form $d$ is symmetric and coercive in $\mathbf{H}$. Let

$$
\mathbf{W}:=\{(\mathbf{u}, \mathbf{v}) \in \mathbf{V}: b((\mathbf{u}, \mathbf{v}), q)=0, \forall q \in Q\}=\{(\mathbf{u}, \mathbf{v}) \in \mathbf{V}: \operatorname{div} \mathbf{u}=0\} ;
$$

Brezzi's conditions for the source problem associated to VP should be:

H1: $a$ is coercive on $\mathbf{W}$, 
H2: $b$ satisfies the inf-sup condition

$$
\inf _{q \in Q}\left[\sup _{\substack{(\mathbf{u}, \mathbf{v}) \in \mathbf{V} \\(\mathbf{u}, \mathbf{v}) \neq(\mathbf{0}, \mathbf{0})}} \frac{b((\mathbf{u}, \mathbf{v}), q)}{\|(\mathbf{u}, \mathbf{v})\||q|}\right] \geq \beta .
$$

The first condition is clearly not satisfied since $\lambda=0$ is an eigenvalue of VP. In fact, for any $\xi \in H_{0}^{1}\left(\Omega_{\mathrm{F}}\right),(\operatorname{curl} \xi, \mathbf{0}) \in \mathbf{W}$ and $a((\operatorname{curl} \xi, \mathbf{0}),(\operatorname{curl} \xi, \mathbf{0}))=0$.

Let us denote $\mathbf{K}:=\left\{(\operatorname{curl} \xi, \mathbf{0}), \xi \in H_{0}^{1}\left(\Omega_{\mathrm{F}}\right)\right\}$ the space of pure rotational motions into the fluid not inducing vibrations in the solid. We show below that $\lambda=0$ is an eigenvalue of problem $\mathbf{V P}$ with eigenspace $\mathbf{K} \times\{0\}$. To prove it we use that the bilinear form $b$ satisfies the inf-sup condition H2. Namely:

Lemma 3.1. There exists a strictly positive constant $\beta$ such that for all $q \in L^{2}\left(\Omega_{\mathrm{F}}\right)$

$$
\sup _{\substack{(\mathbf{u}, \mathbf{v}) \in \mathbf{V} \\(\mathbf{u}, \mathbf{v}) \neq(\mathbf{0}, \mathbf{0})}} \frac{b((\mathbf{u}, \mathbf{v}), q)}{\|(\mathbf{u}, \mathbf{v})\|} \geq \beta|q| .
$$

Proof. Clearly, it is enough to show that, for all $q \in L^{2}\left(\Omega_{\mathrm{F}}\right)$, there exists $(\mathbf{u}, \mathbf{v}) \in \mathbf{V}$ satisfying

$$
\operatorname{div} \mathbf{u}=q \quad \text { in } \Omega_{\mathrm{F}} \quad \text { and } \quad\|(\mathbf{u}, \mathbf{v})\| \leq \mathbf{C}|q|
$$

Let $\Omega:=\Omega_{\mathrm{S}} \cup \bar{\Omega}_{\mathrm{F}}$; let $\tilde{q} \in L^{2}(\Omega)$ be the extension of $q$ obtained by defining

$$
\tilde{q}:=-\frac{1}{\left|\Omega_{\mathrm{S}}\right|} \int_{\Omega_{\mathrm{F}}} q \quad \text { in } \Omega_{\mathrm{S}} .
$$

Therefore, $\tilde{q} \in L_{0}^{2}(\Omega):=\left\{q \in L^{2}(\Omega): \int_{\Omega} q=0\right\}$. Since div is an isomorphism of a subspace of $\left[H_{0}^{1}(\Omega)\right]^{2}$ onto $L_{0}^{2}(\Omega)$ (see $[9]$ ), then there exists $\mathbf{w} \in\left[H_{0}^{1}(\Omega)\right]^{2}$ such that

$$
\operatorname{div} \mathbf{w}=\tilde{q} \quad \text { in } \Omega \quad \text { and } \quad\|\mathbf{w}\|_{\left[H^{1}(\Omega)\right]^{2}} \leq \mathbf{C}\|\tilde{q}\|_{L^{2}(\Omega)},
$$

with $\mathbf{C}$ independent of $q$. Let $\mathbf{u}:=\left.\mathbf{w}\right|_{\Omega_{\mathrm{F}}}$ and $\mathbf{v}:=\left.\mathbf{w}\right|_{\Omega_{\mathrm{S}}}$; hence, $(\mathbf{u}, \mathbf{v}) \in \mathbf{V}$ and it satisfies (3.3).

Now it is simple to characterize the eigenspace of $\lambda=0$.

Theorem 3.1. $\lambda=0$ is an eigenvalue of $\boldsymbol{V P}$ with eigenspace $\mathbf{K} \times\{0\}$.

Proof. $\forall \xi \in H_{0}^{1}\left(\Omega_{\mathrm{F}}\right),(\mathbf{c u r l} \xi, \mathbf{0}, 0)$ is clearly an eigenfunction of $\mathbf{V P}$ with eigenvalue $\lambda=0$. Conversely, let $(\mathbf{u}, \mathbf{v}, p) \in \mathbf{V} \times Q$ be such that

$$
\begin{aligned}
& \int_{\Omega_{\mathrm{S}}} \boldsymbol{\sigma}(\mathbf{v}): \boldsymbol{\varepsilon}(\boldsymbol{\psi})-\int_{\Omega_{\mathrm{F}}} p \operatorname{div} \boldsymbol{\phi}=0, \quad \forall(\boldsymbol{\phi}, \boldsymbol{\psi}) \in \mathbf{V}, \\
&-\int_{\Omega_{\mathrm{F}}} q \operatorname{div} \mathbf{u}-\frac{1}{\rho_{\mathrm{F}} c^{2}} \int_{\Omega_{\mathrm{F}}} p q=0, \quad \forall q \in Q .
\end{aligned}
$$

Hence, $\operatorname{div} \mathbf{u}=-\frac{1}{\rho_{\mathrm{F}} c^{2}} p$ in $\Omega_{\mathrm{F}}$ and then, by using $(\boldsymbol{\phi}, \boldsymbol{\psi})=(\mathbf{u}, \mathbf{v})$ in the first equation, $\int_{\Omega_{\mathrm{S}}} \boldsymbol{\sigma}(\mathbf{v}): \boldsymbol{\varepsilon}(\mathbf{v})+\frac{1}{\rho_{\mathrm{F}} c^{2}} \int_{\Omega_{\mathrm{F}}} p^{2}=0$. Therefore, because of Korn's inequality, $\mathbf{v}=\mathbf{0}$ in $\Omega_{\mathrm{S}}$ and, in the compressible case, $p=0$.

In both cases, $\mathbf{u}$ satisfies $\operatorname{div} \mathbf{u}=0$ in $\Omega_{\mathrm{F}}$ and $\mathbf{u} \cdot \boldsymbol{\nu}=\mathbf{v} \cdot \boldsymbol{\nu}=0$ on $\Gamma_{\mathrm{I}}$. Therefore, there exists $\xi \in H_{0}^{1}\left(\Omega_{\mathrm{F}}\right)$ such that $\mathbf{u}=\operatorname{curl} \xi$ in $\Omega_{\mathrm{F}}$. To conclude the proof in the incompressible case we use Lemma 3.1 to show that $p=0$ since $\int_{\Omega_{\mathrm{F}}} p \operatorname{div} \phi=$ $\int_{\Omega_{\mathrm{S}}} \boldsymbol{\sigma}(\mathbf{v}): \varepsilon(\boldsymbol{\psi})=0$ for all $(\phi, \boldsymbol{\psi}) \in \mathbf{V}$. 
The bilinear form $a$ is not coercive on $\mathbf{W}$. However, $a^{*}:=a+d$ can be used instead of $a$. Let us consider this modified eigenvalue problem:

$\mathbf{V P}^{*}:$ Find $\lambda \in \mathbf{R},(\mathbf{u}, \mathbf{v}, p) \in \mathbf{V} \times Q,(\mathbf{u}, \mathbf{v}, p) \neq(\mathbf{0}, \mathbf{0}, 0)$, such that:

$$
\begin{aligned}
a^{*}((\mathbf{u}, \mathbf{v}),(\boldsymbol{\phi}, \boldsymbol{\psi}))+b((\boldsymbol{\phi}, \boldsymbol{\psi}), p) & =\lambda d((\mathbf{u}, \mathbf{v}),(\boldsymbol{\phi}, \boldsymbol{\psi})), \quad \forall(\boldsymbol{\phi}, \boldsymbol{\psi}) \in \mathbf{V}, \\
b((\mathbf{u}, \mathbf{v}), q)-\frac{1}{\rho_{\mathrm{F}} c^{2}}(p, q) & =0, \quad \forall q \in Q .
\end{aligned}
$$

$\lambda$ is an eigenvalue of $\mathbf{V P}$ if and only if $1+\lambda$ is an eigenvalue of $\mathbf{V P}^{*}$ and the eigenfunctions for both problems coincide. Problem $\mathbf{V P}^{*}$ is easier to deal with than VP because of the following lemma.

Lemma 3.2. $a^{*}$ is coercive on $\{(\mathbf{u}, \mathbf{v}) \in \mathbf{X}: \operatorname{div} \mathbf{u}=0\} \supset \mathbf{W}$.

Proof. For all $(\mathbf{u}, \mathbf{v}) \in \mathbf{X}$ such that $\operatorname{div} \mathbf{u}=0$,

$$
a^{*}((\mathbf{u}, \mathbf{v}),(\mathbf{u}, \mathbf{v}))=\int_{\Omega_{\mathrm{S}}} \boldsymbol{\sigma}(\mathbf{v}): \varepsilon(\mathbf{v})+\int_{\Omega_{\mathrm{F}}} \rho_{\mathrm{F}}|\mathbf{u}|^{2}+\int_{\Omega_{\mathrm{S}}} \rho_{\mathrm{S}}|\mathbf{v}|^{2} \geq \alpha\|(\mathbf{u}, \mathbf{v})\|^{2},
$$

with $\alpha$ being the minimum between $\rho_{\mathrm{F}}$ and the constant in Korn's inequality.

The bilinear forms $a^{*}$ and $b$ satisfy both of Brezzi's conditions. Then, the mixed source problem associated to $\mathbf{V} \mathbf{P}^{*}$ is well-posed. Moreover, we have the following result:

Lemma 3.3. Given $(\mathbf{f}, \mathbf{g}) \in \mathbf{H}, w \in Q$ and $\delta \geq 0$, there exists a unique solution $(\mathbf{u}, \mathbf{v}, p) \in \mathbf{V} \times Q$ of the source problem

$$
\begin{aligned}
a^{*}((\mathbf{u}, \mathbf{v}),(\boldsymbol{\phi}, \boldsymbol{\psi}))+b((\boldsymbol{\phi}, \boldsymbol{\psi}), p) & =d((\mathbf{f}, \mathbf{g}),(\boldsymbol{\phi}, \boldsymbol{\psi})), \quad \forall(\boldsymbol{\phi}, \boldsymbol{\psi}) \in \mathbf{V}, \\
b((\mathbf{u}, \mathbf{v}), q)-\delta(p, q) & =(w, q), \quad \forall q \in Q .
\end{aligned}
$$

Moreover,

$$
\|(\mathbf{u}, \mathbf{v})\|+|p| \leq \mathbf{C}(|(\mathbf{f}, \mathbf{g})|+|w|)
$$

with a constant $\mathbf{C}$ independent of $\delta$.

Proof. See, for instance, [5].

\section{Characterization of the spectrum}

If we consider $w=0$ and $\delta=\frac{1}{\rho_{\mathrm{F}} c^{2}}$ in Lemma 3.3, we obtain the source problem associated to $\mathbf{V P}^{*}$. Namely, to find $(\mathbf{u}, \mathbf{v}, p) \in \mathbf{V} \times Q$ such that

$$
\begin{aligned}
a^{*}((\mathbf{u}, \mathbf{v}),(\boldsymbol{\phi}, \boldsymbol{\psi}))+b((\boldsymbol{\phi}, \boldsymbol{\psi}), p) & =d((\mathbf{f}, \mathbf{g}),(\boldsymbol{\phi}, \boldsymbol{\psi})), \quad \forall(\boldsymbol{\phi}, \boldsymbol{\psi}) \in \mathbf{V}, \\
b((\mathbf{u}, \mathbf{v}), q)-\frac{1}{\rho_{\mathrm{F}} c^{2}}(p, q) & =0, \quad \forall q \in Q .
\end{aligned}
$$

Let

$$
\mathbf{T}: \begin{array}{clc}
\mathbf{H} & \longrightarrow \mathbf{V} \\
(\mathbf{f}, \mathbf{g}) & \longmapsto & (\mathbf{u}, \mathbf{v})
\end{array}
$$

with $(\mathbf{u}, \mathbf{v}, p)$ being the solution of (4.1)-(4.2). Because of (3.5), $\mathbf{T}$ is a bounded operator and its bound does not depend on the acoustic speed $c$ (the bound being valid even for $c=\infty)$.

$(\lambda,(\mathbf{u}, \mathbf{v}))$ is an eigenpair of $\left.\mathbf{T}\right|_{\mathbf{V}}$ if and only if there exists $p \in L^{2}\left(\Omega_{\mathrm{F}}\right)$ such that $\left(\frac{1}{\lambda},(\mathbf{u}, \mathbf{v}, p)\right)$ is a solution of $\mathbf{V} \mathbf{P}^{*}$ and, consequently, $\left(\frac{1}{\lambda}-1,(\mathbf{u}, \mathbf{v}, p)\right)$ a solution of 
VP. Therefore, the knowledge of the spectrum of $\left.\mathbf{T}\right|_{\mathbf{V}}$ gives complete information about the solutions of our original problem.

$\left.\mathbf{T}\right|_{\mathbf{V}}$ is not compact; in fact, $\left.\mathbf{T}\right|_{\mathbf{K}}$ is the identity on the infinite dimensional subspace $\mathbf{K} \subset \mathbf{V}$. However, as we show below, the restriction of $\mathbf{T}$ to the orthogonal complement of $\mathbf{K}$ is compact and this can be used to characterize the spectrum of T.

For any function $\mathbf{f} \in\left[L^{2}\left(\Omega_{\mathrm{F}}\right)\right]^{2}$, we may write a Helmholtz decomposition $\mathbf{f}=\operatorname{curl} \xi+\nabla \varphi$ with $\xi \in H_{0}^{1}\left(\Omega_{\mathrm{F}}\right)$ and $\varphi \in H^{1}\left(\Omega_{\mathrm{F}}\right)$. Therefore, the orthogonal complement of $\mathbf{K}$ in $\mathbf{H}$ is

$$
\mathbf{G}:=\mathbf{K}^{\perp_{\mathrm{H}}}=\left\{(\nabla \varphi, \mathbf{v}), \varphi \in H^{1}\left(\Omega_{\mathrm{F}}\right), \mathbf{v} \in\left[L^{2}\left(\Omega_{\mathrm{S}}\right)\right]^{2}\right\}
$$

and, hence, $\mathbf{K}^{\perp_{\mathrm{V}}}=\mathbf{G} \cap \mathbf{V}$. The following lemma shows that $\mathbf{G} \cap \mathbf{V}$ is invariant for T.

Lemma 4.1. $\mathbf{T}(\mathbf{G}) \subset \mathbf{G} \cap \mathbf{V}$.

Proof. Let $(\mathbf{f}, \mathbf{g}) \in \mathbf{G}$ and let $(\mathbf{u}, \mathbf{v}, p) \in \mathbf{V} \times Q$ be the solution of problem (4.1)(4.2); for any $(\operatorname{curl} \xi, \mathbf{0}) \in \mathbf{K}, b((\operatorname{curl} \xi, \mathbf{0}), p)=0$ and, hence, $((\mathbf{u}, \mathbf{v}),(\operatorname{curl} \xi, \mathbf{0}))$ $=\frac{1}{\rho_{\mathrm{F}}} a^{*}((\mathbf{u}, \mathbf{v}),(\operatorname{curl} \xi, \mathbf{0}))=\frac{1}{\rho_{\mathrm{F}}} d((\mathbf{f}, \mathbf{g}),(\operatorname{curl} \xi, \mathbf{0}))=((\mathbf{f}, \mathbf{g}),(\operatorname{curl} \xi, \mathbf{0}))=0$; therefore, $(\mathbf{u}, \mathbf{v}) \in \mathbf{K}^{\perp} \mathrm{v}=\mathbf{G} \cap \mathbf{V}$.

On the other hand, $\left.\mathbf{T}\right|_{\mathbf{G}}$ is a regularizing operator. In fact we have the following a priori estimate.

Theorem 4.1. There exist constants $s \in\left(\frac{1}{2}, 1\right], t \in(0,1]$ and $\mathbf{C}>0$ (not depending on the acoustic speed $c)$ such that if $(\mathbf{u}, \mathbf{v}, p) \in \mathbf{V} \times Q$ is the solution of problem (4.1)-(4.2) with $(\mathbf{f}, \mathbf{g}) \in \mathbf{G}$, then $\mathbf{u} \in\left[H^{s}\left(\Omega_{\mathrm{F}}\right)\right]^{2}, \mathbf{v} \in\left[H^{1+t}\left(\Omega_{\mathrm{S}}\right)\right]^{2}, p \in H^{1}\left(\Omega_{\mathrm{F}}\right)$ and

$$
\|\mathbf{u}\|_{\left[H^{s}\left(\Omega_{\mathrm{F}}\right)\right]^{2}}+\|\mathbf{v}\|_{\left[H^{1+t}\left(\Omega_{\mathrm{S}}\right)\right]^{2}}+\|p\|_{H^{1}\left(\Omega_{\mathrm{F}}\right)} \leq \mathbf{C}|(\mathbf{f}, \mathbf{g})| .
$$

Proof. Let $(\mathbf{f}, \mathbf{g}) \in \mathbf{G}$ and let $(\mathbf{u}, \mathbf{v}, p) \in \mathbf{V} \times Q$ be the solution of problem (4.1)(4.2). Because of Lemma 4.1, (u, $\mathbf{v}) \in \mathbf{G} \cap \mathbf{V}$. Therefore, there exists $\varphi \in H^{1}\left(\Omega_{\mathrm{F}}\right)$ such that $\mathbf{u}=\nabla \varphi$. Since $(\mathbf{u}, \mathbf{v}) \in \mathbf{V}$ and $\operatorname{div} \mathbf{u}=-\frac{1}{\rho_{\mathrm{F}} c^{2}} p$, then $\varphi$ is a solution of the compatible Neumann problem

$$
\begin{aligned}
\Delta \varphi & =-\frac{1}{\rho_{\mathrm{F}} c^{2}} p, & & \text { in } \Omega_{\mathrm{F}}, \\
\frac{\partial \varphi}{\partial \boldsymbol{\nu}} & =\mathbf{v} \cdot \boldsymbol{\nu}, & & \text { on } \Gamma_{\mathrm{I}} .
\end{aligned}
$$

By using the standard a priori estimate for this Neumann problem (see, for instance, [10]) we know that $\varphi \in H^{1+s}\left(\Omega_{\mathrm{F}}\right)$, where $s=1$, if $\Omega_{\mathrm{F}}$ is convex, and $s=\frac{\pi}{\theta}$, with $\theta$ the biggest reentrant corner of $\Omega_{\mathrm{F}}$, otherwise, and

$$
\begin{aligned}
\|\mathbf{u}\|_{\left[H^{s}\left(\Omega_{\mathrm{F}}\right)\right]^{2}} & =\|\nabla \varphi\|_{\left[H^{s}\left(\Omega_{\mathrm{F}}\right)\right]^{2}} \\
& \leq \mathbf{C}\left[\sum_{j=1}^{J}\|\mathbf{v} \cdot \boldsymbol{\nu}\|_{H^{1 / 2}\left(\Gamma_{j}\right)}+\frac{1}{\rho_{\mathrm{F}} c^{2}}\|p\|_{L^{2}\left(\Omega_{\mathrm{F}}\right)}\right] \leq \mathbf{C}|(\mathbf{f}, \mathbf{g})|,
\end{aligned}
$$

where we have used (3.5) for the last inequality.

On the other hand, by using $\phi \in\left[\mathcal{C}_{0}^{\infty}\left(\Omega_{\mathrm{F}}\right)\right]^{2}$ and $\boldsymbol{\psi}=\mathbf{0}$ in (4.1), it turns out that

$$
\nabla p+\rho_{\mathrm{F}} \mathbf{u}=\rho_{\mathrm{F}} \mathbf{f} .
$$


Hence $p \in H^{1}\left(\Omega_{\mathrm{F}}\right)$ and, because of $(3.5)$,

$$
\|p\|_{H^{1}\left(\Omega_{\mathrm{F}}\right)} \leq \mathbf{C}|(\mathbf{f}, \mathbf{g})| .
$$

Finally, for all $\boldsymbol{\psi} \in\left[H_{\Gamma_{\mathrm{D}}}^{1}\left(\Omega_{\mathrm{S}}\right)\right]^{2}$ there exists $\phi \in H\left(\operatorname{div}, \Omega_{\mathrm{F}}\right)$ such that $(\phi, \psi) \in$ V. Then, by using (4.1) and (4.4), we obtain

$$
\int_{\Omega_{\mathrm{S}}} \boldsymbol{\sigma}(\mathbf{v}): \boldsymbol{\varepsilon}(\boldsymbol{\psi})+\int_{\Omega_{\mathrm{S}}} \rho_{\mathrm{S}} \mathbf{v} \cdot \boldsymbol{\psi}=\int_{\Omega_{\mathrm{S}}} \rho_{\mathrm{S}} \mathbf{g} \cdot \boldsymbol{\psi}+\int_{\Gamma_{\mathrm{I}}} p \boldsymbol{\psi} \cdot \boldsymbol{\nu}, \quad \forall \boldsymbol{\psi} \in\left[H_{\Gamma_{\mathrm{D}}}^{1}\left(\Omega_{\mathrm{S}}\right)\right]^{2} .
$$

Hence, $\mathbf{v}$ is the solution (in the sense of distributions) of the following elasticity problem:

$$
\begin{aligned}
-\operatorname{div}[\boldsymbol{\sigma}(\mathbf{v})]+\rho_{\mathrm{S}} \mathbf{v} & =\rho_{\mathrm{S}} \mathbf{g}, & & \text { in } \Omega_{\mathrm{S}} \\
\boldsymbol{\sigma}(\mathbf{v}) \boldsymbol{\nu} & =-p \boldsymbol{\nu}, & & \text { on } \Gamma_{\mathrm{I}}, \\
\boldsymbol{\sigma}(\mathbf{v}) \mathbf{n} & =\mathbf{0}, & & \text { on } \Gamma_{\mathrm{N}} \\
\mathbf{v} & =\mathbf{0}, & & \text { on } \Gamma_{\mathrm{D}}
\end{aligned}
$$

Therefore, by using the standard a priori estimate for this problem, $\mathbf{v} \in\left[H^{1+t}\left(\Omega_{\mathrm{S}}\right)\right]^{2}$ and

$$
\left.\|\mathbf{v}\|_{\left[H^{1+t}\left(\Omega_{\mathrm{S}}\right)\right]^{2}} \leq \mathbf{C}\left\{\|\mathbf{g}\|_{\left[L^{2}\left(\Omega_{\mathrm{S}}\right)\right.}\right]^{2}+\|p\|_{H^{1 / 2}\left(\Gamma_{\mathrm{I}}\right)}\right\} \leq \mathbf{C}|(\mathbf{f}, \mathbf{g})|
$$

with $t \in(0,1]$ depending on the reentrant corners of $\partial \Omega_{\mathrm{S}}$, on the angles between $\Gamma_{\mathrm{N}}$ and $\Gamma_{\mathrm{D}}$ and on the Lamé coefficients $\lambda_{\mathrm{S}}$ and $\mu_{\mathrm{S}}$ (see [10]).

Finally, notice that the constants $\mathbf{C}$ in (4.3), (4.5) and (4.6) only depend on standard a priori estimates and (3.5), hence they are independent of the acoustic speed $c$.

Now we can give a complete characterization of the eigenpairs of $\left.\mathbf{T}\right|_{\mathbf{V}}$ and hence of the solutions of VP.

Theorem 4.2. The spectrum of $\left.\mathbf{T}\right|_{\mathbf{V}}$ consists of the eigenvalue $\lambda=1$ and a sequence of finite multiplicity eigenvalues $\left\{\lambda_{n}: n \in \mathbf{N}\right\} \subset(0,1)$ converging to 0 . K is the eigenspace of $\lambda=1$ and each eigenvector $\left(\mathbf{u}_{n}, \mathbf{v}_{n}\right)$ associated to an eigenvalue $\lambda_{n}<1$ satisfies curl $\mathbf{u}_{n}=0$.

Proof. Since $\left.\mathbf{T}\right|_{\mathbf{K}}$ is the identity, $\mathbf{K}^{\perp_{\mathrm{V}}}=\mathbf{G} \cap \mathbf{V}$ and, $\mathbf{T}(\mathbf{G} \cap \mathbf{V}) \subset \mathbf{G} \cap \mathbf{V}$ (Lemma 4.1), it is enough to prove that $\left.\mathbf{T}\right|_{\mathbf{G} \cap \mathbf{V}}: \mathbf{G} \cap \mathbf{V} \longrightarrow \mathbf{G} \cap \mathbf{V}$ is compact. Now, because of Theorem 4.1, $\mathbf{T}(\mathbf{G} \cap \mathbf{V}) \subset\left[H^{s}\left(\Omega_{\mathrm{F}}\right)\right]^{2} \times\left[H^{1+t}\left(\Omega_{\mathrm{S}}\right)\right]^{2}$ and the latter is compactly imbedded in $\mathbf{H}$. Therefore, $\left.\mathbf{T}\right|_{\mathbf{G} \cap \mathbf{V}}$ is compact.

As a consequence of the last two theorems we have the following result.

Theorem 4.3. Let $(\mathbf{u}, \mathbf{v}, p)$ be an eigenfunction of problem $\mathbf{V P}^{*}$ with corresponding eigenvalue $\lambda>1$. Then $\mathbf{u} \in\left[H^{s}\left(\Omega_{\mathrm{F}}\right)\right]^{2}, \mathbf{v} \in\left[H^{1+t}\left(\Omega_{\mathrm{S}}\right)\right]^{2}, p \in H^{1+s}\left(\Omega_{\mathrm{F}}\right)$ and

$$
\left.\left.\|\mathbf{u}\|_{\left[H^{s}\left(\Omega_{\mathrm{F}}\right)\right.}\right]^{2}+\|\mathbf{v}\|_{\left[H^{1+t}\left(\Omega_{\mathrm{S}}\right)\right.}\right]^{2}+\|p\|_{H^{1+s}\left(\Omega_{\mathrm{F}}\right)} \leq \mathbf{C}|(\mathbf{u}, \mathbf{v})|
$$

with $s$ and $t$ as in the previous theorem and $\mathbf{C}$ a strictly positive constant independent of $c$.

Proof. Since $\lambda \neq 1$, because of Theorem 4.2, $(\mathbf{u}, \mathbf{v}) \in \mathbf{G}$. Now, $(\mathbf{u}, \mathbf{v}, p)$ is the solution of problem (4.1)-(4.2) with $(\mathbf{f}, \mathbf{g})=\frac{1}{\lambda}(\mathbf{u}, \mathbf{v}) \in \mathbf{G}$. Therefore, Theorem 4.1 applies to this case. Moreover, $\nabla p+\rho_{\mathrm{F}} \mathbf{u}=\lambda \rho_{\mathrm{F}} \mathbf{u}$ and so, because of (4.3), $p \in H^{1+s}\left(\Omega_{\mathrm{F}}\right)$ with $\|p\|_{H^{1+s}\left(\Omega_{\mathrm{F}}\right)} \leq \mathbf{C}|(\mathbf{u}, \mathbf{v})|$. 


\section{Finite element discretization}

In the previous section it was shown that $\left.\mathbf{T}\right|_{\mathbf{G} \cap \mathbf{V}}$ is compact. However, a standard discretization of this operator would require to use finite element spaces of irrotational functions. To avoid it, we will deal with the noncompact operator $\mathbf{T}$ instead.

$\mathbf{T}$ has an infinite dimensional eigenspace $\mathbf{K}$ consisting of pure rotational motions which are not physically relevant since they do not induce vibrations into the structure. However, a suitable numerical approximation should take care of them. Otherwise, spurious modes may appear.

In [1] a discretization which does not present spurious modes is introduced for the case of a compressible fluid. We will show that this method can be successfully used for incompressible fluids too. However, the proofs in [1] are not directly valid in this case since the constants in the estimates therein depend on the acoustic speed; in fact, these constants blow up when $c$ goes to infinity. Alternative proofs with constants not depending on $c$ will be given below.

Let $\left\{\mathcal{T}_{h}\right\}$ be a family of regular triangulations of $\Omega_{\mathrm{F}} \cup \Omega_{\mathrm{S}}$ such that every triangle is completely contained either in $\Omega_{\mathrm{F}}$ or in $\Omega_{\mathrm{S}}$ and such that the end points of $\Gamma_{\mathrm{D}}$ coincide with nodes of the triangulation. For each component of the displacements in the solid we use the standard piecewise linear finite element space

$$
L_{h}\left(\Omega_{\mathrm{S}}\right):=\left\{v \in H^{1}\left(\Omega_{\mathrm{S}}\right):\left.v\right|_{T} \in \mathcal{P}_{1}(T), \forall T \in \mathcal{T}_{h}, T \subset \Omega_{\mathrm{S}}\right\}
$$

and, for the fluid, the Raviart-Thomas space [15]

$$
\mathbf{R}_{h}\left(\Omega_{\mathrm{F}}\right):=\left\{\mathbf{u} \in H\left(\operatorname{div}, \Omega_{\mathrm{F}}\right):\left.\mathbf{u}\right|_{T} \in \mathcal{R}_{0}(T), \forall T \in \mathcal{T}_{h}, T \subset \Omega_{\mathrm{F}}\right\},
$$

where

$$
\mathcal{R}_{0}(T):=\left\{\mathbf{u} \in \mathcal{P}_{1}(T)^{2}: \mathbf{u}(x, y)=(a+b x, c+b y), a, b, c \in \mathbf{R}\right\} .
$$

The degrees of freedom in $\mathbf{R}_{h}\left(\Omega_{\mathrm{F}}\right)$ are the (constant) values of the normal component of $\mathbf{u}$ along each edge of the triangulation. The discrete analogue of $\mathbf{X}$ is

$$
\mathbf{X}_{h}:=\left\{(\mathbf{u}, \mathbf{v}) \in \mathbf{R}_{h}\left(\Omega_{\mathrm{F}}\right) \times\left[L_{h}\left(\Omega_{\mathrm{S}}\right)\right]^{2}:\left.\mathbf{v}\right|_{\Gamma_{\mathrm{D}}}=\mathbf{0}\right\} .
$$

Finally, for the pressures we use the space of piecewise constant functions

$$
Q_{h}:=\left\{p \in L^{2}\left(\Omega_{\mathrm{F}}\right):\left.p\right|_{T} \in \mathcal{P}_{0}(T), \forall T \in \mathcal{T}_{h}, T \subset \Omega_{\mathrm{F}}\right\} .
$$

The conforming finite element spaces $\mathbf{V} \cap \mathbf{X}_{h}$ are not adequate for our problem. In fact, any function of these spaces has constant normal components along each edge $\Gamma_{j}$ of the polygonal interface $\Gamma_{\mathrm{I}}$ and, hence, only functions with this same property could be well approximated. The vibration modes of the physical problem does not have constant normal components along these edges, so we are led to impose a weaker condition than (2.5) to define our discrete spaces. In fact, we use the following ones:

$$
\mathbf{V}_{h}:=\left\{(\mathbf{u}, \mathbf{v}) \in \mathbf{X}_{h}: \int_{\ell}(\mathbf{u} \cdot \boldsymbol{\nu}-\mathbf{v} \cdot \boldsymbol{\nu})=0, \forall \ell \subset \Gamma_{\mathrm{I}}, \ell \text { edge of } T, T \in \mathcal{T}_{h}\right\} .
$$

Let us remark that for $(\mathbf{u}, \mathbf{v}) \in \mathbf{V}_{h}, \mathbf{u} \cdot \boldsymbol{\nu}$ and $\mathbf{v} \cdot \boldsymbol{\nu}$ coincide at the midpoint of each edge $\ell \subset \Gamma_{\text {I }}$ but, in general, they do not coincide on the whole edge. Hence, $\mathbf{V}_{h} \not \subset \mathbf{V}$; that is, our method turns out to be nonconforming.

The theorem below shows that the bilinear forms $a^{*}$ and $b$ satisfy both of Brezzi's conditions on the finite element spaces $\mathbf{V}_{h}$ and $Q_{h}$. 
Theorem 5.1. The bilinear forms $a^{*}$ and $b$ satisfy:

$\mathbf{H} \mathbf{1}_{h}: a^{*}$ is coercive on $\mathbf{W}_{h}:=\left\{(\mathbf{u}, \mathbf{v}) \in \mathbf{V}_{h}: b((\mathbf{u}, \mathbf{v}), q)=0, \forall q \in Q_{h}\right\}$,

$\mathbf{H 2}{ }_{h}$ : There exists $\beta>0$ not depending on $h$ such that

$$
\inf _{q \in Q_{h}}\left[\sup _{\substack{(\mathbf{u}, \mathbf{v}) \in \mathbf{V}_{h} \\(\mathbf{u}, \mathbf{v}) \neq(\mathbf{0}, \mathbf{0})}} \frac{b((\mathbf{u}, \mathbf{v}), q)}{\|(\mathbf{u}, \mathbf{v})\||q|}\right] \geq \beta .
$$

Proof. For all $\mathbf{u} \in \mathbf{R}_{h}\left(\Omega_{\mathrm{F}}\right)$, $\operatorname{div} \mathbf{u} \in Q_{h}$; then $\mathbf{W}_{h}=\left\{(\mathbf{u}, \mathbf{v}) \in \mathbf{V}_{h}: \operatorname{div} \mathbf{u}=0\right\}$. Since $a^{*}$ was proved to be coercive on $\{(\mathbf{u}, \mathbf{v}) \in \mathbf{X}: \operatorname{div} \mathbf{u}=0\} \supset \mathbf{W}_{h}$ (Lemma 3.2), then $\mathbf{H} \mathbf{1}_{h}$ is true.

To prove $\mathbf{H} \mathbf{2}_{h}$, we are going to proceed as in Lemma 3.1 and show that for all $q \in Q_{h}$ there exists $\left(\mathbf{u}_{h}, \mathbf{v}_{h}\right) \in \mathbf{V}_{h}$ satisfying

$$
\operatorname{div} \mathbf{u}_{h}=q \quad \text { in } \Omega_{\mathrm{F}} \quad \text { and } \quad\left\|\left(\mathbf{u}_{h}, \mathbf{v}_{h}\right)\right\| \leq \mathbf{C}|q| .
$$

Since $Q_{h} \subset L^{2}\left(\Omega_{\mathrm{F}}\right)$, let $(\mathbf{u}, \mathbf{v}) \in \mathbf{V}$ be defined as in the proof of Lemma 3.1. Then $(\mathbf{u}, \mathbf{v})$ satisfies (3.3).

Let $\mathbf{v}_{h} \in\left[L_{h}\left(\Omega_{\mathrm{S}}\right)\right]^{2}$ be a Clement's type interpolant of $\mathbf{v}$, vanishing on $\Gamma_{\mathrm{D}} \cup \Gamma_{\mathrm{N}}$ and defined at the nodes $B \in \Gamma_{\mathrm{I}}$ in the following way:

$$
\mathbf{v}_{h}(B):=\frac{1}{\left|\ell_{B}^{-}\right|+\left|\ell_{B}^{+}\right|} \int_{\ell_{B}^{-} \cup \ell_{B}^{+}} \mathbf{v}
$$

where $\ell_{B}^{-}$and $\ell_{B}^{+}$are the two edges on $\Gamma_{\mathrm{I}}$ sharing $B$. Proceeding as in [17] it is simple to prove that $\left.\left.\left\|\mathbf{v}_{h}\right\|_{\left[H^{1}\left(\Omega_{\mathrm{S}}\right)\right.}\right]^{2} \leq \mathbf{C}\|\mathbf{v}\|_{\left[H^{1}\left(\Omega_{\mathrm{S}}\right)\right.}\right]^{2}$, and hence, because of (3.3), $\left\|\mathbf{v}_{h}\right\|_{\left[H^{1}\left(\Omega_{\mathrm{S}}\right)\right]^{2}} \leq \mathbf{C}|q|$.

On the other hand, by construction, $\int_{\Gamma_{\mathrm{I}}} \mathbf{v}_{h}=\int_{\Gamma_{\mathrm{I}}} \mathbf{v}$ and, hence, $\int_{\Gamma_{\mathrm{I}}} \mathbf{v}_{h} \cdot \boldsymbol{\nu}=$ $\int_{\Gamma_{\mathrm{I}}} \mathbf{v} \cdot \boldsymbol{\nu}=\int_{\Gamma_{\mathrm{I}}} \mathbf{u} \cdot \boldsymbol{\nu}=\int_{\Omega_{\mathrm{F}}} \operatorname{div} \mathbf{u}=\int_{\Omega_{\mathrm{F}}} q$. Therefore, the following Neumann problem is compatible,

$$
\begin{aligned}
\Delta \varphi & =q, & & \text { in } \Omega_{\mathrm{F}}, \\
\frac{\partial \varphi}{\partial \boldsymbol{\nu}} & =\mathbf{v}_{h} \cdot \boldsymbol{\nu}, & & \text { on } \Gamma_{\mathrm{I}}
\end{aligned}
$$

and, so, it has a unique solution $\varphi \in H^{1}\left(\Omega_{\mathrm{F}}\right) / \mathcal{P}_{0}$. Because of the usual a priori estimate, $\varphi \in H^{1+s}\left(\Omega_{\mathrm{F}}\right)$ with $s$ as in Theorem 4.1 and

$$
\|\nabla \varphi\|_{\left[H^{s}\left(\Omega_{\mathrm{F}}\right)\right]^{2}} \leq \mathbf{C}\left[|q|+\sum_{j=1}^{J}\left\|\mathbf{v}_{h} \cdot \boldsymbol{\nu}\right\|_{H^{1 / 2}\left(\Gamma_{j}\right)}\right] \leq \mathbf{C}|q| .
$$

Let $\mathbf{u}_{h} \in \mathbf{R}_{h}\left(\Omega_{\mathrm{F}}\right)$ be the standard Raviart-Thomas interpolant of $\nabla \varphi$ (see [15]). For each edge $\ell \subset \Gamma_{\mathrm{I}}, \int_{\ell} \mathbf{u}_{h} \cdot \boldsymbol{\nu}=\int_{\ell} \frac{\partial \varphi}{\partial \boldsymbol{\nu}}=\int_{\ell} \mathbf{v}_{h} \cdot \boldsymbol{\nu}$; hence, $\left(\mathbf{u}_{h}, \mathbf{v}_{h}\right) \in \mathbf{V}_{h}$. On the other hand, $\operatorname{div} \mathbf{u}_{h}$ is the $L^{2}\left(\Omega_{\mathrm{F}}\right)$ projection of $\operatorname{div}(\nabla \varphi)=q$ onto $Q_{h}$; since $q \in Q_{h}$, then $\operatorname{div} \mathbf{u}_{h}=q$. Finally, by the stability property of this interpolation, $\left\|\mathbf{u}_{h}\right\|_{H\left(\operatorname{div}, \Omega_{\mathrm{F}}\right)} \leq \mathbf{C}\|\nabla \varphi\|_{H^{1+s}\left(\Omega_{\mathrm{F}}\right)} \leq \mathbf{C}|q|$. Therefore, $\left(\mathbf{u}_{h}, \mathbf{v}_{h}\right) \in \mathbf{V}_{h}$ satisfies (5.1).

By applying the standard theory of mixed methods, we have an analogous result to Lemma 3.3 for the discrete problem. 
Lemma 5.1. Given $(\mathbf{f}, \mathbf{g}) \in \mathbf{H}, w \in Q$ and $\delta \geq 0$, there exists a unique solution $\left(\mathbf{u}_{h}, \mathbf{v}_{h}, p_{h}\right) \in \mathbf{V}_{h} \times Q_{h}$ of the source problem

$$
\begin{aligned}
a^{*}\left(\left(\mathbf{u}_{h}, \mathbf{v}_{h}\right),(\boldsymbol{\phi}, \boldsymbol{\psi})\right)+b\left((\boldsymbol{\phi}, \boldsymbol{\psi}), p_{h}\right) & =d((\mathbf{f}, \mathbf{g}),(\boldsymbol{\phi}, \boldsymbol{\psi})), \quad \forall(\boldsymbol{\phi}, \boldsymbol{\psi}) \in \mathbf{V}_{h}, \\
b\left(\left(\mathbf{u}_{h}, \mathbf{v}_{h}\right), q\right)-\delta\left(p_{h}, q\right) & =(w, q), \quad \forall q \in Q_{h} .
\end{aligned}
$$

Moreover,

$$
\left\|\left(\mathbf{u}_{h}, \mathbf{v}_{h}\right)\right\|+\left|p_{h}\right| \leq \mathbf{C}(|(\mathbf{f}, \mathbf{g})|+|w|),
$$

with a constant $\mathbf{C}$ independent of $\delta$ and $h$.

Proof. See, for instance, [5].

Now, by considering $w=0$ and $\delta=\frac{1}{\rho_{\mathrm{F}} c^{2}}$ we can define a discrete analogue of $\mathbf{T}$. Namely $\mathbf{T}_{h}: \mathbf{H} \longrightarrow \mathbf{V}_{h}$ where $\left(\mathbf{u}_{h}, \mathbf{v}_{h}\right)=\mathbf{T}_{h}(\mathbf{f}, \mathbf{g})$ is such that there exists $p_{h} \in Q_{h}$ satisfying

$$
\begin{aligned}
a^{*}\left(\left(\mathbf{u}_{h}, \mathbf{v}_{h}\right),(\boldsymbol{\phi}, \boldsymbol{\psi})\right)+b\left((\boldsymbol{\phi}, \boldsymbol{\psi}), p_{h}\right) & =d((\mathbf{f}, \mathbf{g}),(\boldsymbol{\phi}, \boldsymbol{\psi})), \quad \forall(\boldsymbol{\phi}, \boldsymbol{\psi}) \in \mathbf{V}_{h}, \\
b\left(\left(\mathbf{u}_{h}, \mathbf{v}_{h}\right), q\right)-\frac{1}{\rho_{\mathrm{F}} c^{2}}\left(p_{h}, q\right) & =0, \quad \forall q \in Q_{h} .
\end{aligned}
$$

Because of (5.2), the operators $\mathbf{T}_{h}$ are bounded uniformly on $h$ and $c$. The spectra of these operators provide good approximations of the spectrum of $\mathbf{T}$ as is shown in the next section.

In other words, we approximate the solutions of problem $\mathbf{V P}^{*}$ by means of its discrete analogue:

$\mathbf{V P}_{h}^{*}:$ Find $\lambda_{h} \in \mathbf{R},\left(\mathbf{u}_{h}, \mathbf{v}_{h}, p_{h}\right) \in \mathbf{V}_{h} \times Q_{h},\left(\mathbf{u}_{h}, \mathbf{v}_{h}, p_{h}\right) \neq(\mathbf{0}, \mathbf{0}, 0)$, such that:

$$
\begin{aligned}
a^{*}\left(\left(\mathbf{u}_{h}, \mathbf{v}_{h}\right),(\boldsymbol{\phi}, \boldsymbol{\psi})\right)+b\left((\boldsymbol{\phi}, \boldsymbol{\psi}), p_{h}\right) & =\lambda_{h} d\left(\left(\mathbf{u}_{h}, \mathbf{v}_{h}\right),(\boldsymbol{\phi}, \boldsymbol{\psi})\right), \quad \forall(\boldsymbol{\phi}, \boldsymbol{\psi}) \in \mathbf{V}_{h} \\
b\left(\left(\mathbf{u}_{h}, \mathbf{v}_{h}\right), q\right)-\frac{1}{\rho_{\mathrm{F}} c^{2}}\left(p_{h}, q\right) & =0, \quad \forall q \in Q_{h} .
\end{aligned}
$$

\section{Spectral approximation}

In order to show that the eigenvalues and eigenfuctions of $\left.\mathbf{T}\right|_{\mathbf{V}}$ can be well approximated by those of $\left.\mathbf{T}_{h}\right|_{\mathbf{V}_{h}}$ we are going to use the theory developed in [7] for noncompact operators as it is used (in the case of a compressible fluid) in [1].

Throughout this section we write $\sigma(\cdot)$ to denote the spectrum of an operator.

Since $\mathbf{V}_{h} \subset \mathbf{X}, \mathbf{T}_{h}$ can be seen as a conforming discretization of the operator $\left.\mathbf{T}\right|_{\mathbf{X}}: \mathbf{X} \longrightarrow \mathbf{X}$. On the other hand, the knowledge of the spectrum of $\left.\mathbf{T}\right|_{\mathbf{X}}$ gives complete information about the spectrum of $\left.\mathbf{T}\right|_{\mathbf{V}}$. In fact, $\sigma\left(\left.\mathbf{T}\right|_{\mathbf{X}}\right)=\sigma\left(\left.\mathbf{T}\right|_{\mathbf{V}}\right) \cup\{0\}$ (see $[1])$.

Two properties have to be proved to apply the theory in [7]. The first one (P1) means that the finite element spaces used are adequate to approximate the physically relevant eigenfunctions of $\mathbf{T}$. The second one (P2) means that $\mathbf{T}_{h}$ provides good approximations of $\mathbf{T}$ when applied to sources $(\mathbf{f}, \mathbf{g})$ in the discrete space. Both properties will be shown to be valid uniformly on $c$.

On the other hand, the discrete operators $\mathbf{T}_{h}$ have eigenspaces providing good approximations of the infinite dimensional eigenspace $\mathbf{K}$ of $\mathbf{T}$ with exactly the same eigenvalue. 
Theorem 6.1. $\lambda=1$ is an eigenvalue of $\mathbf{T}_{h}$ with corresponding eigenspace $\mathbf{K}_{h}=$ $\mathbf{K} \cap \mathbf{V}_{h}=\left\{(\operatorname{curl} \xi, \mathbf{0}), \xi \in L_{h}\left(\Omega_{\mathrm{F}}\right):\left.\xi\right|_{\Gamma_{\mathrm{I}}}=0\right\}$.

Proof. The proof is omitted since it is essentially the same as that of Theorem 4.2 in [1].

A $\mathbf{V}_{h}$-interpolant is introduced in [1] and the following approximation property is proved:

Lemma 6.1. There exist a linear operator $\mathbf{I}_{h}: \mathbf{V} \cap\left\{\left[H^{s}\left(\Omega_{\mathrm{F}}\right)\right]^{2} \times\left[H^{1+t}\left(\Omega_{\mathrm{S}}\right)\right]^{2}\right\} \rightarrow$ $\mathbf{V}_{h}$ and a strictly positive constant $\mathbf{C}$ such that, if $\operatorname{div} \mathbf{u} \in H^{1}\left(\Omega_{\mathrm{F}}\right)$, then

$$
\left.\left\|(\mathbf{u}, \mathbf{v})-\mathbf{I}_{h}(\mathbf{u}, \mathbf{v})\right\| \leq \mathbf{C} h^{r}\left\{\|\mathbf{u}\|_{\left[H^{s}\left(\Omega_{\mathrm{F}}\right)\right.}\right]^{2}+\|\operatorname{div} \mathbf{u}\|_{H^{1}\left(\Omega_{\mathrm{F}}\right)}+\|\mathbf{v}\|_{\left[H^{1+t}\left(\Omega_{\mathrm{S}}\right)\right]^{2}}\right\},
$$

where $r:=\min \{s, t\}$ with $s \in\left(\frac{1}{2}, 1\right]$ and $t \in(0,1]$ as in Theorem 4.1.

Proof. See Theorem 5.2 in [1].

Property $\mathbf{P} 1$ is a simple consequence of the previous lemma.

Theorem 6.2. (P1) For each eigenfunction $(\mathbf{u}, \mathbf{v})$ of $\mathbf{T}$ associated to an eigenvalue $\lambda \in(0,1)$, with $\|(\mathbf{u}, \mathbf{v})\|=1$, there exists a strictly positive constant $\mathbf{C}$, depending neither on $h$ nor on $c$, such that

$$
\operatorname{dist}\left((\mathbf{u}, \mathbf{v}), \mathbf{V}_{h}\right) \leq \mathbf{C} h^{r},
$$

where dist is the distance measured in the norm $\|\cdot\|$ and $r:=\min \{s, t\}$ as in the previous lemma.

Proof. Being $\mathbf{T}(\mathbf{u}, \mathbf{v})=\lambda(\mathbf{u}, \mathbf{v})$, there exists $p \in L^{2}\left(\Omega_{\mathrm{F}}\right)$ such that $(\mathbf{u}, \mathbf{v}, p)$ is an eigenfunction of problem $\mathbf{V P}^{*}$ with eigenvalue $\frac{1}{\lambda}>1$. Hence, since $\|(\mathbf{u}, \mathbf{v})\|=1$, by using Theorem 4.3, we have

$$
\left.\|\mathbf{u}\|_{\left[H^{s}\left(\Omega_{\mathrm{F}}\right)\right.}\right]^{2}+\|\mathbf{v}\|_{\left[H^{1+t}\left(\Omega_{\mathrm{S}}\right)\right]^{2}}+\|p\|_{H^{1}\left(\Omega_{\mathrm{F}}\right)} \leq \mathbf{C}|(\mathbf{u}, \mathbf{v})| \leq \mathbf{C},
$$

with $\mathbf{C}$ depending on $\lambda$ but not on $c$. Moreover, since $\operatorname{div} \mathbf{u}=\frac{1}{\rho_{\mathrm{F}} c^{2}} p$, then, for any $c \geq c_{0}$ (finite or infinite), $\|\operatorname{div} \mathbf{u}\|_{H^{1}\left(\Omega_{\mathrm{F}}\right)} \leq \mathbf{C}$. So, by applying Lemma 6.1 , we have

$$
\operatorname{dist}\left((\mathbf{u}, \mathbf{v}), \mathbf{V}_{h}\right) \leq\left\|(\mathbf{u}, \mathbf{v})-\mathbf{I}_{h}(\mathbf{u}, \mathbf{v})\right\| \leq \mathbf{C} h^{r} .
$$

To prove $\mathbf{P 2}$ we need to modify the proofs in [1] in order to obtain a result independent of the acoustic speed, valid even for $c=\infty$.

Theorem 6.3. (P2) There exists a strictly positive constant $\mathbf{C}$, depending neither on $h$ nor on $c$, such that, for all $(\mathbf{f}, \mathbf{g}) \in \mathbf{V}_{h}$,

$$
\left\|\left(\mathbf{T}-\mathbf{T}_{h}\right)(\mathbf{f}, \mathbf{g})\right\| \leq \mathbf{C} h^{r}\|(\mathbf{f}, \mathbf{g})\|,
$$

with $r=\min \{s, t\}$ as above.

Proof. Since $\mathbf{T}$ and $\mathbf{T}_{h}$ coincide on $\mathbf{K}_{h}$, it is enough to prove the theorem for $(\mathbf{f}, \mathbf{g}) \in \mathbf{K}_{h}^{\perp} \mathrm{V}_{h}$. So, let $(\mathbf{f}, \mathbf{g}) \in \mathbf{K}_{h}^{\perp_{\mathrm{V}_{h}}}$; since $(\mathbf{f}, \mathbf{g}) \in \mathbf{V}_{h}$, then the following Neumann problem is compatible:

$$
\begin{aligned}
\Delta \varphi & =\operatorname{div} \mathbf{f}, & & \text { in } \Omega_{\mathrm{F}}, \\
\frac{\partial \varphi}{\partial \boldsymbol{\nu}} & =\mathrm{g} \cdot \boldsymbol{\nu}, & & \text { on } \Gamma_{\mathrm{I}} .
\end{aligned}
$$


Let $\varphi$ be a solution of this problem; because of the standard a priori estimate, $\varphi \in \mathbf{H}^{1+s}\left(\Omega_{\mathrm{F}}\right)$ and $\|\nabla \varphi\|_{\left[H^{s}\left(\Omega_{\mathrm{F}}\right)\right]^{2}} \leq \mathbf{C}\|(\mathbf{f}, \mathbf{g})\|$. Now, $\operatorname{div}(\mathbf{f}-\nabla \varphi)=0$ and $\int_{\Gamma_{\mathrm{I}}}(\mathbf{f}-\nabla \varphi) \cdot \boldsymbol{\nu}=0$; therefore, there exists $\zeta \in H^{1}\left(\Omega_{\mathrm{F}}\right)$ such that

$$
(\mathbf{f}, \mathbf{g})=(\operatorname{curl} \zeta, \mathbf{0})+(\nabla \varphi, \mathbf{g}) .
$$

Since $\mathbf{T}$ and $\mathbf{T}_{h}$ are bounded uniformly on $c$ (and $\mathbf{T}_{h}$ on $h$ too),

$$
\left.\left\|\left(\mathbf{T}-\mathbf{T}_{h}\right)(\operatorname{curl} \zeta, \mathbf{0})\right\| \leq\left(\|\mathbf{T}\|+\left\|\mathbf{T}_{h}\right\|\right)\|(\operatorname{curl} \zeta, \mathbf{0})\| \leq \mathbf{C}|\operatorname{curl} \zeta|_{\left[L^{2}\left(\Omega_{\mathrm{F}}\right)\right.}\right]^{2}
$$

Now, $|\operatorname{curl} \zeta|_{\left[L^{2}\left(\Omega_{\mathrm{F}}\right)\right]^{2}} \leq \mathbf{C} h^{s}\|\nabla \varphi\|_{\left[H^{s}\left(\Omega_{\mathrm{F}}\right)\right]^{2}}$ (see Lemma 5.5 of $\left.[1]\right)$; hence,

$$
\left\|\left(\mathbf{T}-\mathbf{T}_{h}\right)(\operatorname{curl} \zeta, \mathbf{0})\right\| \leq \mathbf{C} h^{s}\|(\mathbf{f}, \mathbf{g})\| .
$$

On the other hand, let $(\mathbf{u}, \mathbf{v}):=\mathbf{T}(\nabla \varphi, \mathbf{g})$; let $p \in Q$ be such that $(\mathbf{u}, \mathbf{v}, p)$ is the solution of the mixed problem (4.1)-(4.2) with $\mathbf{f}$ substituted by $\nabla \varphi$. Analogously, let $\left(\mathbf{u}_{h}, \mathbf{v}_{h}\right):=\mathbf{T}_{h}(\nabla \varphi, \mathbf{g})$ and $p_{h} \in Q_{h}$ such that $\left(\mathbf{u}_{h}, \mathbf{v}_{h}, p_{h}\right)$ is the solution of the discrete problem (5.3)-(5.4) with $\mathbf{f}$ again substituted by $\nabla \varphi$. That is, $\left(\mathbf{u}_{h}, \mathbf{v}_{h}, p_{h}\right)$ is the finite element approximate solution of (4.1)-(4.2) on $\mathbf{V}_{h} \times Q_{h}$. Since $\mathbf{V}_{h}$ is not a subspace of $\mathbf{V}$, it is a nonconforming approximation; however, the standard theory can be easily adapted to cover this case.

Following the lines of [5] (Sections II.2.4 and II.2.6), by using that $\mathbf{V}_{h}$ and $Q_{h}$ satisfy both of Brezzi's conditions (Theorem 5.1), it is easy to show that

$$
\begin{gathered}
\left\|(\mathbf{u}, \mathbf{v})-\left(\mathbf{u}_{h}, \mathbf{v}_{h}\right)\right\| \leq \mathbf{C}\left[\operatorname{dist}\left((\mathbf{u}, \mathbf{v}), \mathbf{V}_{h}\right)+\operatorname{dist}\left(p, Q_{h}\right)\right. \\
\left.+\sup _{\substack{\phi, \psi) \in \mathbf{V}_{h} \\
(\phi, \psi) \neq(\mathbf{0}, \mathbf{0})}} \frac{a^{*}((\mathbf{u}, \mathbf{v}),(\boldsymbol{\phi}, \boldsymbol{\psi}))+b(p,(\boldsymbol{\phi}, \boldsymbol{\psi}))-d((\nabla \varphi, \mathbf{g}),(\boldsymbol{\phi}, \boldsymbol{\psi}))}{\|(\boldsymbol{\phi}, \boldsymbol{\psi})\|}\right],
\end{gathered}
$$

with a constant $\mathbf{C}$ depending neither on $c$ nor on $h$.

Since $(\nabla \varphi, \mathbf{g}) \in \mathbf{G}$, by virtue of Theorem 4.1 ,

$$
\left.\|\mathbf{u}\|_{\left[H^{s}\left(\Omega_{\mathrm{F}}\right)\right]^{2 ،}}+\|\mathbf{v}\|_{\left[H^{1+t}\left(\Omega_{\mathrm{S}}\right)\right.}\right]^{2}+\|p\|_{H^{1}\left(\Omega_{\mathrm{F}}\right)} \leq \mathbf{C}|(\nabla \varphi, \mathbf{g})|
$$

(C independent of $c$ ); hence,

$$
\operatorname{dist}\left(p, Q_{h}\right) \leq \mathbf{C} h\|p\|_{H^{1}\left(\Omega_{\mathrm{F}}\right)} \leq \mathbf{C} h|(\nabla \varphi, \mathbf{g})|
$$

and, by using Lemma 6.1 as in Theorem 6.2,

$$
\operatorname{dist}\left((\mathbf{u}, \mathbf{v}), \mathbf{V}_{h}\right) \leq \mathbf{C} h^{r}|(\nabla \varphi, \mathbf{g})| \text {. }
$$

The remaining consistency term in the right hand side of (6.3) can be bounded proceeding as in Lemma 5.7 of [1]; by so doing, we obtain for all $(\boldsymbol{\phi}, \boldsymbol{\psi}) \in \mathbf{V}_{h}$

$$
\begin{aligned}
\left|a^{*}((\mathbf{u}, \mathbf{v}),(\boldsymbol{\phi}, \boldsymbol{\psi}))+b(p,(\boldsymbol{\phi}, \boldsymbol{\psi}))-d((\nabla \boldsymbol{\varphi}, \mathbf{g}),(\boldsymbol{\phi}, \boldsymbol{\psi}))\right| & =\left|\int_{\Gamma_{\mathrm{I}}} p(\boldsymbol{\phi} \cdot \boldsymbol{\nu}-\boldsymbol{\psi} \cdot \boldsymbol{\nu})\right| \\
& \leq \mathbf{C} h|p|_{H^{1}\left(\Omega_{\mathrm{F}}\right)}\|(\boldsymbol{\phi}, \boldsymbol{\psi})\| \\
& \leq \mathbf{C} h|(\nabla \varphi, \mathbf{g})|\|(\boldsymbol{\phi}, \boldsymbol{\psi})\| .
\end{aligned}
$$

Therefore, by using (6.4), (6.5) and (6.6) in (6.3), we obtain

$$
\left\|\left(\mathbf{T}-\mathbf{T}_{h}\right)(\nabla \varphi, \mathbf{g})\right\|=\left\|(\mathbf{u}, \mathbf{v})-\left(\mathbf{u}_{h}, \mathbf{v}_{h}\right)\right\| \leq \mathbf{C} h^{r}|(\nabla \varphi, \mathbf{g})| \leq \mathbf{C} h^{r}|(\mathbf{f}, \mathbf{g})|,
$$


which, together with (6.2) and (6.1), allow us to conclude the theorem.

Once properties $\mathbf{P 1}$ and $\mathbf{P 2}$ have been proved, we may apply the spectral approximation theory of [7] as it was used in [1] for a compressible fluid. The next theorem shows that there are no spurious eigenvalues for $h$ small enough.

Theorem 6.4. Let $J$ be a closed interval such that $J \cap \sigma(\mathbf{T})=\emptyset$. There exists a strictly positive constant $h_{J}$ such that if $h \leq h_{J}$, then $J \cap \sigma\left(\mathbf{T}_{h}\right)=\emptyset$.

For an open interval $I \subset(0,1)$, let $\mathbf{E}_{I}$ be the direct sum of the eigenspaces of $\mathbf{T}$ associated with its eigenvalues in $I$. Let us denote by $\mathbf{E}_{I}^{h}$ the analogue for $\mathbf{T}_{h}$. We have the following error estimates for approximate eigenmodes.

Theorem 6.5. There exist strictly positive constants $\mathbf{C}$ and $h_{I}$ such that, if $h \leq$ $h_{I}$, then

1. for each $\left(\mathbf{u}_{h}, \mathbf{v}_{h}\right) \in \mathbf{E}_{I}^{h}$ with $\left\|\left(\mathbf{u}_{h}, \mathbf{v}_{h}\right)\right\|=1$, dist $\left(\left(\mathbf{u}_{h}, \mathbf{v}_{h}\right), \mathbf{E}_{I}\right) \leq \mathbf{C} h^{r}$;

2. for each $(\mathbf{u}, \mathbf{v}) \in \mathbf{E}_{I}$ with $\|(\mathbf{u}, \mathbf{v})\|=1$, $\operatorname{dist}\left((\mathbf{u}, \mathbf{v}), \mathbf{E}_{I}^{h}\right) \leq \mathbf{C} h^{r}$;

in both cases $r:=\min \{s, t\}$ as above.

As a consequence of this theorem, if $I \cap \sigma(\mathbf{T})=\{\lambda\}$, with $\lambda \in(0,1)$, then, for $h$ small enough, the dimension of the linear space $\mathbf{E}_{I}^{h}$ must coincide with that of $\mathbf{E}_{I}$ (let us say $n$ ). This implies the convergence to $\lambda$ of exactly $n$ eigenvalues of the discrete problem $\lambda_{h}^{(1)} \ldots \lambda_{h}^{(n)}$. Moreover, the following error estimate with a double order of convergence has been proved in [14]:

Theorem 6.6. There exist strictly positive constants $\mathbf{C}$ and $h_{I}$ such that if $h \leq h_{I}$ then

$$
\left|\lambda-\lambda_{h}^{(i)}\right| \leq \mathbf{C} h^{2 r}, \quad i=1, \ldots, n,
$$

with $r$ as above and $\mathbf{C}$ depending on $\lambda$.

This theorem implies that the eigenvalues $\lambda>1$ of $\mathbf{V P}^{*}$ are approximated with order $h^{2 r}$ by as many eigenvalues of $\mathbf{V P}_{h}^{*}$ (repeated according to their multiplicities) as the multiplicity of $\lambda$. On the other hand, Theorem 6.4 shows that all the eigenvalues of $\mathbf{V P}_{h}^{*}$ are approximations of eigenvalues of $\mathbf{V P} \mathbf{P}^{*}$. Theorems 3.1 and 6.1 show that $\lambda=1$ is an eigenvalue of both problems with corresponding eigenspaces $\mathbf{K} \times\{0\}$ and $\mathbf{K}_{h} \times\{0\}$ respectively. Moreover, $\mathbf{V P}_{h}^{*}$ does not have any other eigenvalue $\lambda_{h} \neq 1$ converging to $\lambda=1$. In fact, if $\left(\lambda_{h},\left(\mathbf{u}_{h}, \mathbf{v}_{h}, p_{h}\right)\right)$ is an eigenpair of $\mathbf{V P}_{h}^{*}$ with $\left(\mathbf{u}_{h}, \mathbf{v}_{h}\right) \in \mathbf{K}_{h}^{\perp_{\mathrm{v}_{h}}}$, then $\lambda_{h} \geq 1+\delta$ with $\delta>0$ independent of $h$ and $c$. To prove this, it is enough to show that

$$
\int_{\Omega_{\mathrm{S}}} \boldsymbol{\sigma}\left(\mathbf{v}_{h}\right): \varepsilon\left(\mathbf{v}_{h}\right)+\frac{1}{\rho_{\mathrm{F}} c^{2}} \int_{\Omega_{\mathrm{F}}} p_{h}^{2} \geq \delta\left(\int_{\Omega_{\mathrm{F}}} \rho_{\mathrm{F}}\left|\mathbf{u}_{h}\right|^{2}+\int_{\Omega_{\mathrm{S}}} \rho_{\mathrm{S}}\left|\mathbf{v}_{h}\right|^{2}\right)
$$

(in fact, if (6.7) is true, then by using $(\boldsymbol{\phi}, \boldsymbol{\psi}, q)=\left(\mathbf{u}_{h}, \mathbf{v}_{h}, p_{h}\right)$ in $\mathbf{V P}_{h}^{*}$ it turns out that $\left.\lambda_{h} \geq 1+\delta\right)$. To prove (6.7) we split $\left(\mathbf{u}_{h}, \mathbf{v}_{h}\right)$ as in (6.1): $\left(\mathbf{u}_{h}, \mathbf{v}_{h}\right)=$ $(\operatorname{curl} \zeta, \mathbf{0})+\left(\nabla \varphi, \mathbf{v}_{h}\right)$, again with $\left(\nabla \varphi, \mathbf{v}_{h}\right) \in \mathbf{V}$. Proceeding as in Theorem 6.3 we have

$$
\begin{aligned}
\left\|\left(\mathbf{u}_{h}, \mathbf{v}_{h}\right)\right\| & \left.\leq|\operatorname{curl} \zeta|_{\left[L^{2}\left(\Omega_{\mathrm{F}}\right)\right.}\right]^{2}+\left\|\left(\nabla \varphi, \mathbf{v}_{h}\right)\right\| \\
& \left.\leq \mathbf{C} h^{s}\left\|\left(\mathbf{u}_{h}, \mathbf{v}_{h}\right)\right\|+\mathbf{C}\left\{\left|\operatorname{div} \mathbf{u}_{h}\right|+\left\|\mathbf{v}_{h}\right\|_{\left[H^{1}\left(\Omega_{\mathrm{F}}\right)\right.}\right]^{2}\right\}
\end{aligned}
$$


Hence, for $h$ small enough, $\left\|\left(\mathbf{u}_{h}, \mathbf{v}_{h}\right)\right\| \leq \mathbf{C}\left\{\left|\operatorname{div} \mathbf{u}_{h}\right|+\left\|\mathbf{v}_{h}\right\|_{\left[H^{1}\left(\Omega_{\mathrm{F}}\right)\right]^{2}}\right\}$ and so (6.7) is a consequence of this inequality, Korn's inequality and the fact that div $\mathbf{u}_{h}=$ $\frac{1}{\rho_{\mathrm{F}} c^{2}} p_{h}$.

Now, let $\lambda_{1} \leq \lambda_{2} \leq \ldots \leq \lambda_{n} \leq \ldots$ be all the eigenvalues of $\mathbf{V P}^{*}$ (counted by their multiplicities) with irrotational corresponding eigenmodes (i.e., those $\lambda_{n}>1$; see Theorem 4.2). Let $\lambda_{h 1} \leq \lambda_{h 2} \leq \cdots \leq \lambda_{h N_{h}}$ be all the eigenvalues of $\mathbf{V P}_{h}^{*}$ strictly greater than 1 (counted by their multiplicities too). Then, for all $j \in \mathbf{N}$,

$$
\left|\lambda_{h j}-\lambda_{j}\right| \leq \mathbf{C} h^{2 r}
$$

with $h$ small enough as to have $N_{h} \geq j$ and $\mathbf{C}$ depending on $\lambda_{j}$ but neither on $h$ nor on $c \in\left[c_{0}, \infty\right]$.

\section{Asymptotic BehaVior FOR $c \rightarrow \infty$}

The vibration problem for an incompressible fluid is a limit case of that for a compressible one. In this section we show that the eigenfrequencies in the first case are actually limits of those in the second case as $c$ goes to infinity. This result is true for the continuous problem as well as for the discretization analyzed above.

The results in this section show that it is possible to deal with a nearly incompressible fluid by considering it as perfectly incompressible. An advantage of such a treatment is that less degrees of freedom are necessary to discretize the fluid in the last case. In fact, in the next section, we show that, in the incompressible case, a stream function can be used instead of the displacements into the fluid in order to save computational effort.

To analyze the asympotic behavior of our problem, we are going to use the arguments in [8]. In that paper a different problem is considered: the Stokes one and a regularized (or penalized) version of it. However, the arguments therein are general as it can be easily verified; indeed their proofs are based on the min-max principle and estimates like (7.1) and (7.2) below for the source problem.

Throughout this section we use an index $c$ in order to remark explicitly the dependence of certain magnitudes on the acoustic speed $c \in\left[c_{0}, \infty\right]$. For instance, for a given $(\mathbf{f}, \mathbf{g}) \in \mathbf{H}$, let $\left(\mathbf{u}^{c}, \mathbf{v}^{c}, p^{c}\right)$ be the solution of the source problem (4.1)-(4.2) and $\left(\mathbf{u}_{h}^{c}, \mathbf{v}_{h}^{c}, p_{h}^{c}\right)$ that of the corresponding discrete problem (5.3)-(5.4). Because of (5.2), we know that these solutions are uniformly bounded with respect to $h$ and $c$, that is,

$$
\left\|\left(\mathbf{u}_{h}^{c}, \mathbf{v}_{h}^{c}\right)\right\| \leq \mathbf{C}|(\mathbf{f}, \mathbf{g})| .
$$

On the other hand, since the discrete source problem is well-posed (Lemma 5.1), then it is immediate to show that the solutions of (5.3)-(5.4) for finite $c$ converge to that for $c=\infty$ with an error of order $\frac{1}{c^{2}}$. More precisely, we have

$$
\left\|\left(\mathbf{u}_{h}^{c}, \mathbf{v}_{h}^{c}\right)-\left(\mathbf{u}_{h}^{\infty}, \mathbf{v}_{h}^{\infty}\right)\right\| \leq \frac{\mathbf{C}}{c^{2}}|(\mathbf{f}, \mathbf{g})|
$$

The number of solutions of the discrete problem for the incompressible case is smaller than that for the compressible one. To show this, we rewrite the mixed problem $\mathbf{V P}_{h}^{*}$ as a true eigenvalue problem. We need to distinguish between the compressible and the incompressible cases. In the first one, by using (5.6), the pressure can be eliminated in (5.5) obtaining the following variational problem: 
$\mathbf{C P}_{h}:$ Find $\lambda_{h}^{c} \in \mathbf{R},\left(\mathbf{u}_{h}^{c}, \mathbf{v}_{h}^{c}\right) \in \mathbf{V}_{h},\left(\mathbf{u}_{h}^{c}, \mathbf{v}_{h}^{c}\right) \neq(\mathbf{0}, \mathbf{0})$, such that:

$a^{*}\left(\left(\mathbf{u}_{h}^{c}, \mathbf{v}_{h}^{c}\right),(\boldsymbol{\phi}, \boldsymbol{\psi})\right)+\rho_{\mathrm{F}} c^{2} \int_{\Omega_{\mathrm{F}}} \operatorname{div} \mathbf{u}_{h}^{c} \operatorname{div} \boldsymbol{\phi}=\lambda_{h}^{c} d\left(\left(\mathbf{u}_{h}^{c}, \mathbf{v}_{h}^{c}\right),(\boldsymbol{\phi}, \boldsymbol{\psi})\right)$,

$$
\forall(\phi, \psi) \in \mathbf{V}_{h} .
$$

$\mathbf{C P}_{h}$ is equivalent to $\mathbf{V} \mathbf{P}_{h}^{*}$ for $c<\infty$. In fact, clearly any solution of $\mathbf{V} \mathbf{P}_{h}^{*}$ gives a solution of $\mathbf{C P}_{h}$. Conversely, if $\left(\lambda_{h}^{c},\left(\mathbf{u}_{h}^{c}, \mathbf{v}_{h}^{c}\right)\right)$ is an eigenpair of $\mathbf{C P}_{h}$, then $\left(\lambda_{h}^{c},\left(\mathbf{u}_{h}^{c}, \mathbf{v}_{h}^{c}, \rho_{\mathrm{F}} c^{2} \operatorname{div} \mathbf{u}_{h}^{c}\right)\right)$ is an eigenpair of $\mathbf{V} \mathbf{P}_{h}^{*}$.

In the incompressible case, instead, (5.6) implies that $\operatorname{div} \mathbf{u}_{h}^{\infty}=0$ in $\Omega_{\mathrm{F}}$ and hence the solutions can be found in the subspace $\mathbf{W}_{h}:=\left\{(\mathbf{u}, \mathbf{v}) \in \mathbf{V}_{h}: \operatorname{div} \mathbf{u}=0\right\}$. Therefore, in this case, we have the following problem:

IP $\mathbf{P}_{h}:$ Find $\lambda_{h}^{\infty} \in \mathbf{R},\left(\mathbf{u}_{h}^{\infty}, \mathbf{v}_{h}^{\infty}\right) \in \mathbf{W}_{h},\left(\mathbf{u}_{h}^{\infty}, \mathbf{v}_{h}^{\infty}\right) \neq(\mathbf{0}, \mathbf{0})$, such that:

$$
a^{*}\left(\left(\mathbf{u}_{h}^{\infty}, \mathbf{v}_{h}^{\infty}\right),(\boldsymbol{\phi}, \boldsymbol{\psi})\right)=\lambda_{h}^{\infty} d\left(\left(\mathbf{u}_{h}^{\infty}, \mathbf{v}_{h}^{\infty}\right),(\boldsymbol{\phi}, \boldsymbol{\psi})\right), \quad \forall(\boldsymbol{\phi}, \boldsymbol{\psi}) \in \mathbf{W}_{h} .
$$

$\mathbf{I P}{ }_{h}$ is equivalent to $\mathbf{V P} \mathbf{P}_{h}^{*}$ for $c=\infty$. In fact, clearly, any solution of $\mathbf{V P}_{h}^{*}$ gives a solution of $\mathbf{I P}{ }_{h}$. Conversely, given an eigenpair $\left(\lambda_{h}^{\infty},\left(\mathbf{u}_{h}^{\infty}, \mathbf{v}_{h}^{\infty}\right)\right)$ of $\mathbf{I P}$, since $b$ satisfies the inf-sup condition $\mathbf{H 2} \mathbf{2}_{h}$, then there exists $p_{h}^{\infty} \in Q_{h}$ such that $\left(\lambda_{h}^{\infty},\left(\mathbf{u}_{h}^{\infty}, \mathbf{v}_{h}^{\infty}, p_{h}^{\infty}\right)\right)$ is an eigenpair of $\mathbf{V} \mathbf{P}_{h}^{*}$ (see [9]).

Now, let $M_{h}$ and $N_{h}$ be the number of eigenvalues of $\mathbf{V P}_{h}^{*}$ (counted by their multiplicities) for a compressible and for an incompressible fluid respectively. Because of the previous analysis, these numbers are equal to the dimensions of $\mathbf{V}_{h}$ and $\mathbf{W}_{h}$ respectively. Since $\mathbf{W}_{h} \subset \mathbf{V}_{h}$, then $N_{h} \leq M_{h}$.

In both cases, the smallest eigenvalue is $\lambda_{h}=1$ with the same multiplicity; in fact, its eigenspace is $\mathbf{K}_{h} \times\{0\}$ independently of $c$ (Theorem 6.1). The other eigenvalues of the incompressible problem are approximated by those of the compressible one. In fact, let $M_{h}^{\prime}:=M_{h}-\operatorname{dim}\left(\mathbf{K}_{h}\right)$ and $N_{h}^{\prime}:=N_{h}-\operatorname{dim}\left(\mathbf{K}_{h}\right)$; let $\lambda_{h 1}^{c} \leq \lambda_{h 2}^{c} \leq \cdots \leq \lambda_{h M_{h}^{\prime}}^{c}$ and $\lambda_{h 1}^{\infty} \leq \lambda_{h 2}^{\infty} \leq \cdots \leq \lambda_{h N_{h}^{\prime}}^{\infty}$ be all the eigenvalues strictly greater than one in each case. We have the following result:

Theorem 7.1. For any $j \in \mathbf{N}$, there exist strictly positive constants $h^{*}, c^{*}$ and $\mathbf{C}$ such that if $h \leq h^{*}$ and $c \geq c^{*}$, then

$$
\left|\lambda_{h j}^{c}-\lambda_{h j}^{\infty}\right| \leq \frac{\mathbf{C}}{c^{2}}
$$

with $\mathbf{C}$ independent of $h$ and $c$.

Proof. The arguments in [8] can be followed exactly in our case. Indeed, the proofs therein are based on estimates like (7.1) and (7.2) and on the fact that $\left|\lambda_{h j}^{\infty}-\lambda_{j}^{\infty}\right| \rightarrow$ 0 as $h \rightarrow 0$, with $\lambda_{j}^{\infty}$ being the corresponding eigenvalue of the continuous problem VP ${ }^{*}$ which, in our case, is a consequence of (6.8).

A similar result is valid for the continuous problem. Also in this case, for finite or infinite values of $c$, the smallest eigenvalue is $\lambda=1$ with the infinite dimensional eigenspace $\mathbf{K} \times\{0\}$ (Theorem 3.1). Let $\lambda_{1}^{c} \leq \lambda_{2}^{c} \leq \ldots \leq \lambda_{n}^{c} \leq \ldots$ be all the eigenvalues of $\mathbf{V P}^{*}$ (counted by their multiplicities), such that $\lambda_{n}^{c}>1$. We have the following result:

Theorem 7.2. For any $j \in \mathbf{N}$, there exist strictly positive constants $c^{*}$ and $\mathbf{C}$ such that if $c \geq c^{*}$, then

$$
\left|\lambda_{j}^{c}-\lambda_{j}^{\infty}\right| \leq \frac{\mathbf{C}}{c^{2}}
$$

with $\mathbf{C}$ independent of $h$ and $c$. 
Proof. By adding and subtracting $\lambda_{h j}^{c}$ and $\lambda_{h j}^{\infty}$ and by using Theorem 7.1 and (6.8) we conclude the theorem by passing to the limit as $h \rightarrow 0$.

In the discrete as well as in the continuous case, the problem with a compressible fluid has eigenfrequencies $\omega_{c}=\sqrt{\lambda^{c}}$ which do not converge to those of the incompressible one as the acoustic speed goes to infinity. These additional eigenfrequencies correspond to vibration modes which, in the case of a compressible fluid into a perfectly rigid cavity, are exactly proportional to $c$. In the case of an elastic solid it has been experimentally observed (see [2]) that there are eigenfrequencies in the discrete compressible problem which blow up like $c$ as $c \rightarrow \infty$.

We can characterize these high eigenfrequencies by using formal asymptotic expansions. Let $\lambda^{c} \in \mathbf{R}$ and $\left(\mathbf{u}^{c}, \mathbf{v}^{c}, p^{c}\right) \in \mathbf{V}$ be a solution of $\mathbf{V P}$. By eliminating $p^{c}$ into (3.1) by means of (3.2) we have that

$$
\begin{aligned}
& \frac{1}{c^{2}} \int_{\Omega_{\mathrm{S}}} \boldsymbol{\sigma}\left(\mathbf{v}^{c}\right): \varepsilon(\boldsymbol{\psi})+\int_{\Omega_{\mathrm{F}}} \rho_{\mathrm{F}} \operatorname{div} \mathbf{u}^{c} \operatorname{div} \boldsymbol{\phi} \\
& \quad=\delta^{c}\left(\int_{\Omega_{\mathrm{F}}} \rho_{\mathrm{F}} \mathbf{u}^{c} \cdot \boldsymbol{\phi}+\int_{\Omega_{\mathrm{S}}} \rho_{\mathrm{S}} \mathbf{v}^{c} \cdot \boldsymbol{\psi}\right), \quad \forall(\boldsymbol{\phi}, \boldsymbol{\psi}) \in \mathbf{V},
\end{aligned}
$$

with $\delta^{c}:=\frac{\lambda^{c}}{c^{2}}$. Let us assume that there are asymptotic expansions in powers of $\frac{1}{c^{2}}$ of the form

$$
\begin{aligned}
\left(\mathbf{u}^{c}, \mathbf{v}^{c}\right) & =\left(\mathbf{u}_{0}, \mathbf{v}_{0}\right)+\frac{1}{c^{2}}\left(\mathbf{u}_{1}, \mathbf{v}_{1}\right)+\frac{1}{c^{4}}\left(\mathbf{u}_{2}, \mathbf{v}_{2}\right)+\cdots, \\
\delta^{c} & =\delta_{0}+\frac{1}{c^{2}} \delta_{1}+\frac{1}{c^{4}} \delta_{2}+\cdots .
\end{aligned}
$$

(An attempt to do this rigorously could be made by following the techniques in [16]; for instance, such analysis has been made in [13] for the case of a solid surrounded by a fluid.)

By replacing (7.4) and (7.5) into (7.3) and equating the $0^{\text {th }}$ order terms we obtain:

$$
\int_{\Omega_{\mathrm{F}}} \rho_{\mathrm{F}} \operatorname{div} \mathbf{u}_{0} \operatorname{div} \boldsymbol{\phi}=\delta_{0}\left(\int_{\Omega_{\mathrm{F}}} \rho_{\mathrm{F}} \mathbf{u}_{0} \cdot \boldsymbol{\phi}+\int_{\Omega_{\mathrm{S}}} \rho_{\mathrm{S}} \mathbf{v}_{0} \cdot \boldsymbol{\psi}\right), \quad \forall(\boldsymbol{\phi}, \boldsymbol{\psi}) \in \mathbf{V} .
$$

Now $\lambda^{c}=c^{2} \delta_{0}+\delta_{1}+\frac{1}{c^{2}} \delta_{2}+\cdots$ is one of the eigenvalues blowing up with $c$ if and only if $\delta_{0} \neq 0$. In this case, by taking $\psi \in \mathcal{C}_{0}^{\infty}\left(\Omega_{\mathrm{S}}\right)$ and $\phi=0$, we deduce that $\mathbf{v}_{0}=0$. Therefore,

$$
\int_{\Omega_{\mathrm{F}}} \operatorname{div} \mathbf{u}_{0} \operatorname{div} \phi=\delta_{0} \int_{\Omega_{\mathrm{F}}} \mathbf{u}_{0} \cdot \phi
$$

for all $\phi \in H\left(\operatorname{div}, \Omega_{\mathrm{F}}\right)$ such that there exists $\boldsymbol{\psi} \in\left[H^{1}\left(\Omega_{\mathrm{S}}\right)\right]^{2}$ with $(\phi, \psi) \in \mathbf{V}$; in particular, for all $\phi \in\left[H^{1}\left(\Omega_{\mathrm{F}}\right)\right]^{2}$. Hence (7.6) is equivalent to

$$
\begin{aligned}
-\nabla\left(\operatorname{div} \mathbf{u}_{0}\right) & =\delta_{0} \mathbf{u}_{0}, \quad \operatorname{in} \Omega_{\mathrm{F}}, \\
\operatorname{div} \mathbf{u}_{0} & =0, \quad \text { on } \Gamma_{\mathrm{I}},
\end{aligned}
$$

and the latter is equivalent to the eigenvalue problem for the Laplace operator with 
homogeneous Dirichlet boundary conditions,

$$
\begin{aligned}
-\Delta q & =\delta_{0} q, \quad \text { in } \Omega_{\mathrm{F}}, \\
q & =0, \quad \text { on } \Gamma_{\mathrm{I}},
\end{aligned}
$$

with $q=\operatorname{div} \mathbf{u}_{0}$.

Therefore, assuming the asymptotic expansions (7.4) and (7.5) to be valid, we have the following characterization for the eigenvalues blowing up with $c$ for the compressible fluid

$$
\lambda^{c}=c^{2} \delta_{0}+\delta_{1}+\frac{1}{c^{2}} \delta_{2}+\cdots
$$

with $\delta_{0}$ any eigenvalue of the Dirichlet problem (7.7)-(7.8). These eigenvalues do not appear in the case of an incompressible fluid.

Notice that (7.3) is a singular perturbation spectral problem since, as $c$ goes to infinity, the integral over the solid domain in the right hand side disappears. From a physical point of view this means that the solid become softer and softer and, in the limit, the whole interface $\Gamma_{\mathrm{I}}$ would be free for the fluid; that is, the kinematic condition $\mathbf{u} \cdot \boldsymbol{\nu}=\mathbf{v} \cdot \boldsymbol{\nu}$ would be lost and $p=0$ on $\Gamma_{\mathrm{I}}$ would be the boundary condition instead. Thus, for very large $c$, the problem has a boundary layer at the interface allowing for this kinematic condition to hold. The numerical experiments confirm such a behavior for the high eigenfrequencies of the discrete compressible problem.

\section{The INCOMPRESSIBLE CASE}

In the previous section, it was shown how the pressure can be eliminated in $\mathbf{V} \mathbf{P}_{h}^{*}$. Two alternative discrete problems have been obtained: $\mathbf{C P}_{h}$ for a compressible fluid and $\mathbf{I P}{ }_{h}$ for an incompressible one. Problem $\mathbf{C} \mathbf{P}_{h}$ has been analyzed in [1] and numerical experiments with this problem have been reported in [3]. In this section we describe how to solve problem $\mathbf{I P}_{h}$. We also show how to impose efficiently the interface conditions and how to compute the pressure into the fluid for each eigenmode.

Let us remark that for any $(\mathbf{u}, \mathbf{v}) \in \mathbf{W}_{h}$, the fluid displacement $\mathbf{u}$ is the curl of a piecewise linear continuous stream function in $\Omega_{\mathrm{F}}$. In fact, it is immediate to verify that $\left\{\mathbf{u} \in \mathbf{R}_{h}\left(\Omega_{\mathrm{F}}\right): \operatorname{div} \mathbf{u}=0\right\}=\left\{\operatorname{curl} \xi: \xi \in L_{h}\left(\Omega_{\mathrm{F}}\right)\right\}$. Therefore, problem IP $h$ can be thought of as a piecewise linear continuous discretization of a stream function formulation of our problem for an incompressible fluid.

Much less degrees of freedom are necessary to represent the fluid displacements in this way. In fact, the dimension of $\left\{\operatorname{curl} \xi: \xi \in L_{h}\left(\Omega_{\mathrm{F}}\right)\right\}$ is equal to the number of vertices of $\mathcal{T}_{h}$ in $\bar{\Omega}_{F}$ minus 1 whereas the dimension of $\mathbf{R}_{h}\left(\Omega_{\mathrm{F}}\right)$ is equal to the respective number of edges.

However, by using this discrete stream function formulation, it is more complicated to impose the interface conditions. When using Raviart-Thomas elements for the fluid this was quite simple since the constant values of $\left.\mathbf{u} \cdot \boldsymbol{\nu}\right|_{\ell}$ for $\ell \subset \Gamma_{\mathrm{I}}$ could be substituted by the average of $\left.\mathbf{v} \cdot \boldsymbol{\nu}\right|_{\ell}$ at both vertices of $\ell$. Instead, now, there is no local way of imposing this interface condition.

To avoid dealing with global constraints we have used the following hybridization process. Let $\mathbf{Y}_{h}:=\left\{(\operatorname{curl} \xi, \mathbf{v}): \xi \in L_{h}\left(\Omega_{\mathrm{F}}\right), \mathbf{v} \in\left[L_{h}\left(\Omega_{\mathrm{S}}\right)\right]^{2},\left.\mathbf{v}\right|_{\Gamma_{\mathrm{D}}}=\mathbf{0}\right\}$ be the subspace of $\mathbf{X}_{h}$ with divergence free displacements into the fluid. Let $P_{h}:=\{\mu \in$ $\left.L^{2}\left(\Gamma_{\mathrm{I}}\right):\left.\mu\right|_{\ell} \in \mathcal{P}_{0}(\ell), \forall \ell \subset \Gamma_{\mathrm{I}}\right\}$. Let us consider the hybrid problem: 
$\mathbf{H P}_{h}:$ Find $\lambda_{h} \in \mathbf{R},\left(\mathbf{u}_{h}, \mathbf{v}_{h}, \mu_{h}\right) \in \mathbf{Y}_{h} \times P_{h},\left(\mathbf{u}_{h}, \mathbf{v}_{h}, \mu_{h}\right) \neq(\mathbf{0}, \mathbf{0}, 0)$, such that:

$$
\begin{array}{r}
a^{*}\left(\left(\mathbf{u}_{h}, \mathbf{v}_{h}\right),(\boldsymbol{\phi}, \boldsymbol{\psi})\right)+\int_{\Gamma_{\mathrm{I}}} \mu_{h}(\boldsymbol{\phi} \cdot \boldsymbol{\nu}-\boldsymbol{\psi} \cdot \boldsymbol{\nu})=\lambda_{h} d\left(\left(\mathbf{u}_{h}, \mathbf{v}_{h}\right),(\boldsymbol{\phi}, \boldsymbol{\psi})\right), \\
\forall(\boldsymbol{\phi}, \boldsymbol{\psi}) \in \mathbf{Y}_{h}, \\
\int_{\Gamma_{\mathrm{I}}} \zeta\left(\mathbf{u}_{h} \cdot \boldsymbol{\nu}-\mathbf{v}_{h} \cdot \boldsymbol{\nu}\right)=0, \forall \zeta \in P_{h} .
\end{array}
$$

Problems $\mathbf{I P}{ }_{h}$ and $\mathbf{H P}{ }_{h}$ are equivalent. In fact,

$$
\mathbf{W}_{h}=\left\{(\boldsymbol{\phi}, \boldsymbol{\psi}) \in \mathbf{Y}_{h}: \int_{\Gamma_{\mathrm{I}}} \zeta(\boldsymbol{\phi} \cdot \boldsymbol{\nu}-\boldsymbol{\psi} \cdot \boldsymbol{\nu})=0, \forall \zeta \in P_{h}\right\}
$$

and hence any solution of $\mathbf{H P}_{h}$ gives a solution of $\mathbf{I P}_{h}$. The converse is also true. Let $\left(\lambda_{h},\left(\mathbf{u}_{h}, \mathbf{v}_{h}, \mu_{h}\right)\right)$ be an eigenpair of $\mathbf{I P}_{h}$. As it is shown below (Lemma 8.2) the bilinear forms in problem $\mathbf{H P}_{h}$ satisfy both of Brezzi's conditions; hence there exists a unique solution $\left(\tilde{\mathbf{u}}_{h}, \tilde{\mathbf{v}}_{h}, \mu_{h}\right)$ of the mixed source problem

$$
\begin{aligned}
a^{*}\left(\left(\tilde{\mathbf{u}}_{h}, \tilde{\mathbf{v}}_{h}\right),(\boldsymbol{\phi}, \boldsymbol{\psi})\right)+\int_{\Gamma_{\mathrm{I}}} \mu_{h}(\boldsymbol{\phi} \cdot \boldsymbol{\nu}-\boldsymbol{\psi} \cdot \boldsymbol{\nu})= & \lambda_{h} d\left(\left(\mathbf{u}_{h}, \mathbf{v}_{h}\right),(\boldsymbol{\phi}, \boldsymbol{\psi})\right), \\
\int_{\Gamma_{\mathrm{I}}} \zeta\left(\tilde{\mathbf{u}}_{h} \cdot \boldsymbol{\nu}-\tilde{\mathbf{v}}_{h} \cdot \boldsymbol{\nu}\right)= & \quad \forall(\boldsymbol{\phi}, \boldsymbol{\psi}) \in \mathbf{Y}_{h}, \quad \forall \zeta \in P_{h} .
\end{aligned}
$$

Hence, $\left(\tilde{\mathbf{u}}_{h}, \tilde{\mathbf{v}}_{h}\right) \in \mathbf{W}_{h}$ and it satisfies $a^{*}\left(\left(\tilde{\mathbf{u}}_{h}, \tilde{\mathbf{v}}_{h}\right),(\boldsymbol{\phi}, \boldsymbol{\psi})\right)=\lambda_{h} d\left(\left(\mathbf{u}_{h}, \mathbf{v}_{h}\right),(\boldsymbol{\phi}, \boldsymbol{\psi})\right)$, for all $(\boldsymbol{\phi}, \boldsymbol{\psi}) \in \mathbf{W}_{h}$. Since the only solution of this problem is $\left(\mathbf{u}_{h}, \mathbf{v}_{h}\right)$, then $\left(\lambda_{h},\left(\mathbf{u}_{h}, \mathbf{v}_{h}, \mu_{h}\right)\right)$ is an eigenpair of $\mathbf{H P}_{h}$.

Since problems $\mathbf{I P}{ }_{h}$ and $\mathbf{H} \mathbf{P}_{h}$ are equivalent, the latter may be conveniently solved to compute the approximate eigenvalues of our original problem. On the other hand, we show below that $\mu_{h}$ is an approximation of the pressure of the fluid at the interface $\left.p\right|_{\Gamma_{\mathrm{I}}}$. Notice that $\mathbf{H P}_{h}$ can also be considered as a conforming discretization of the following variational eigenvalue problem:

HP: Find $\lambda \in \mathbf{R},(\mathbf{u}, \mathbf{v}, \mu) \in \mathbf{Y} \times P,(\mathbf{u}, \mathbf{v}, \mu) \neq(\mathbf{0}, \mathbf{0}, 0)$, such that:

$$
\begin{aligned}
a^{*}((\mathbf{u}, \mathbf{v}),(\boldsymbol{\phi}, \boldsymbol{\psi}))+ & \int_{\Gamma_{\mathrm{I}}} \mu(\boldsymbol{\phi} \cdot \boldsymbol{\nu}-\boldsymbol{\psi} \cdot \boldsymbol{\nu})=\lambda d((\mathbf{u}, \mathbf{v}),(\boldsymbol{\phi}, \boldsymbol{\psi})), \quad \forall(\boldsymbol{\phi}, \boldsymbol{\psi}) \in \mathbf{Y}, \\
& \int_{\Gamma_{\mathrm{I}}} \zeta(\mathbf{u} \cdot \boldsymbol{\nu}-\mathbf{v} \cdot \boldsymbol{\nu})=0, \quad \forall \zeta \in P,
\end{aligned}
$$

where $P:=L^{2}\left(\Gamma_{\mathrm{I}}\right)$ and $\mathbf{Y}:=\left\{(\mathbf{u}, \mathbf{v}) \in \mathbf{X}: \operatorname{div} \mathbf{u}=0 \operatorname{in} \Omega_{\mathrm{F}}\right.$ and $\left.\left.\mathbf{u} \cdot \boldsymbol{\nu}\right|_{\Gamma_{\mathrm{I}}} \in L^{2}\left(\Gamma_{\mathrm{I}}\right)\right\}$ with the norm $\|(\mathbf{u}, \mathbf{v})\|_{\mathbf{Y}}^{2}:=\|(\mathbf{u}, \mathbf{v})\|^{2}+\|\mathbf{u} \cdot \boldsymbol{\nu}\|_{L^{2}\left(\Gamma_{\mathrm{I}}\right)}^{2}$.

The continuous bilinear forms $a^{*}$ on $\mathbf{Y} \times \mathbf{Y}$ and $\int_{\Gamma_{\mathrm{I}}} \zeta(\mathbf{u} \cdot \boldsymbol{\nu}-\mathbf{v} \cdot \boldsymbol{\nu})$ on $\mathbf{Y} \times P$ satisfy both of Brezzi's conditions:

Lemma 8.1. (i) $a^{*}$ is coercive on $\left\{(\mathbf{u}, \mathbf{v}) \in \mathbf{Y}: \int_{\Gamma_{\mathrm{I}}} \zeta(\mathbf{u} \cdot \boldsymbol{\nu}-\mathbf{v} \cdot \boldsymbol{\nu})=0, \forall \zeta \in P\right\}$.

(ii) There exists $\beta>0$ such that

$$
\sup _{\substack{(\mathbf{u}, \mathbf{v}) \in \mathbf{Y} \\(\mathbf{u}, \mathbf{v}) \neq(\mathbf{0}, \mathbf{0})}} \frac{\int_{\Gamma_{\mathrm{I}}} \zeta(\mathbf{u} \cdot \boldsymbol{\nu}-\mathbf{v} \cdot \boldsymbol{\nu})}{\|(\mathbf{u}, \mathbf{v})\|_{\mathbf{Y}}} \geq \beta\|\zeta\|_{L^{2}\left(\Gamma_{\mathrm{I}}\right)}, \quad \forall \zeta \in P .
$$


Proof. Since $\left\{(\mathbf{u}, \mathbf{v}) \in \mathbf{Y}: \int_{\Gamma_{I}} \zeta(\mathbf{u} \cdot \boldsymbol{\nu}-\mathbf{v} \cdot \boldsymbol{\nu})=0, \forall \zeta \in P\right\}=\mathbf{W}$, (i) is a consequence of Lemma 3.2 and the fact that, for all $(\mathbf{u}, \mathbf{v}) \in \mathbf{W},\|\mathbf{u} \cdot \boldsymbol{\nu}\|_{L^{2}\left(\Gamma_{\mathrm{I}}\right)}=$ $\|\mathbf{v} \cdot \boldsymbol{\nu}\|_{L^{2}\left(\Gamma_{\mathrm{I}}\right)} \leq \mathbf{C}\|\mathbf{v}\|_{\left[H^{1}\left(\Omega_{\mathrm{S}}\right)\right]^{2}} \leq\|(\mathbf{u}, \mathbf{v})\|$.

To prove (ii) we are going to show that for each $\zeta \in P=L^{2}\left(\Gamma_{\mathrm{I}}\right)$, there exists $(\mathbf{u}, \mathbf{v}) \in \mathbf{Y}$ such that

$$
\left.(\mathbf{u} \cdot \boldsymbol{\nu}-\mathbf{v} \cdot \boldsymbol{\nu})=\zeta \quad \text { and } \quad\|(\mathbf{u}, \mathbf{v})\|_{\mathbf{Y}} \leq \mathbf{C}\|\zeta\|_{L^{2}\left(\Gamma_{\mathrm{I}}\right.}\right) \cdot
$$

Let $\zeta \in L^{2}\left(\Gamma_{\mathrm{I}}\right)$. Since the normal trace operator is onto $H^{-1 / 2}\left(\Gamma_{\mathrm{I}}\right)$, then there exists $\boldsymbol{\phi} \in H\left(\operatorname{div}, \Omega_{\mathrm{F}}\right)$ such that $\boldsymbol{\phi} \cdot \boldsymbol{\nu}=-\zeta$ and $\|\boldsymbol{\phi}\|_{H\left(\operatorname{div}, \Omega_{\mathrm{F}}\right)} \leq \mathbf{C}\|\zeta\|_{L^{2}\left(\Gamma_{\mathrm{I}}\right)}$. Let $q=\operatorname{div} \phi$ and $\tilde{q}$ be its extension to $\Omega:=\Omega_{\mathrm{S}} \cup \bar{\Omega}_{\mathrm{F}}$ as in (3.4). Let $\mathbf{w} \in\left[H_{0}^{1}(\Omega)\right]^{2}$ be as defined in Lemma 3.1; that is,

$$
\operatorname{div} \mathbf{w}=\tilde{q} \text { in } \Omega \quad \text { and } \quad\|\mathbf{w}\|_{\left[H^{1}(\Omega)\right]^{2}} \leq \mathbf{C}\|\tilde{q}\|_{L^{2}(\Omega)} \leq \mathbf{C}\|\operatorname{div} \phi\|_{L^{2}\left(\Omega_{\mathrm{F}}\right)} .
$$

Let $\mathbf{u}:=\left.\mathbf{w}\right|_{\Omega_{\mathrm{F}}}-\boldsymbol{\phi}$ and $\mathbf{v}:=\left.\mathbf{w}\right|_{\Omega_{\mathrm{S}}} ;$ then $(\mathbf{u}, \mathbf{v}) \in \mathbf{Y},(\mathbf{u} \cdot \boldsymbol{\nu}-\mathbf{v} \cdot \boldsymbol{\nu})=\zeta$ and $\|(\mathbf{u}, \mathbf{v})\|_{\mathbf{Y}} \leq \mathbf{C}\|\zeta\|_{L^{2}\left(\Gamma_{\mathrm{I}}\right)}$.

By using the previous lemma, problem $\mathbf{H P}$ can be shown to be equivalent to $\mathbf{V P}^{*}$ for $c=\infty$, with $\mu=\left.p\right|_{\Gamma_{\mathrm{I}}}$ being the pressure of the fluid at the interface. Since this pressure is harmonic in $\Omega_{\mathrm{F}}$, it can be recovered from these boundary values. We are going to show that, for each eigenpair of $\mathbf{H P}_{h}, \mu_{h} \in P_{h}$ gives an approximation of that pressure at the interface that can be used to effectively compute it into the fluid.

First we show that the bilinear forms $a^{*}$ and $\int_{\Gamma_{I}} \zeta(\mathbf{u} \cdot \boldsymbol{\nu}-\mathbf{v} \cdot \boldsymbol{\nu})$ satisfy both of Brezzi's conditions on the discrete spaces $\mathbf{Y}_{h}$ and $P_{h}$ uniformly on $h$ :

Lemma 8.2. (i) $a^{*}$ is coercive on $\left\{(\mathbf{u}, \mathbf{v}) \in \mathbf{Y}_{h}: \int_{\Gamma_{I}} \zeta(\mathbf{u} \cdot \boldsymbol{\nu}-\mathbf{v} \cdot \boldsymbol{\nu})=0, \forall \zeta \in P_{h}\right\}$.

(ii) There exists $\beta>0$ (independent of $h$ ) such that

$$
\sup _{\substack{(\mathbf{u}, \mathbf{v}) \in \mathbf{Y} h \\(\mathbf{u}, \mathbf{v}) \neq(\mathbf{0}, \mathbf{0})}} \frac{\int_{\Gamma_{\mathrm{I}}} \zeta(\mathbf{u} \cdot \boldsymbol{\nu}-\mathbf{v} \cdot \boldsymbol{\nu})}{\|(\mathbf{u}, \mathbf{v})\|_{\mathbf{Y}}} \geq \beta\|\zeta\|_{L^{2}\left(\Gamma_{\mathrm{I}}\right)}, \quad \forall \zeta \in P_{h} .
$$

Proof. Since $\left\{(\mathbf{u}, \mathbf{v}) \in \mathbf{Y}_{h}: \int_{\Gamma_{\mathrm{I}}} \zeta(\mathbf{u} \cdot \boldsymbol{\nu}-\mathbf{v} \cdot \boldsymbol{\nu})=0, \forall \zeta \in P_{h}\right\}=\mathbf{W}_{h}$, (i) is a consequence of Theorem 5.1 and the fact that, for all $(\mathbf{u}, \mathbf{v}) \in \mathbf{W}_{h},\left.\mathbf{u} \cdot \boldsymbol{\nu}\right|_{\Gamma_{\mathrm{I}}}$ is the $L^{2}\left(\Gamma_{\mathrm{I}}\right)$ projection of $\left.\mathbf{v} \cdot \boldsymbol{\nu}\right|_{\Gamma_{\mathrm{I}}}$ onto $P_{h}$ and, hence, $\|\mathbf{u} \cdot \boldsymbol{\nu}\|_{L^{2}\left(\Gamma_{\mathrm{I}}\right)} \leq\|\mathbf{v} \cdot \boldsymbol{\nu}\|_{L^{2}\left(\Gamma_{\mathrm{I}}\right)} \leq$ $\mathbf{C}\|\mathbf{v}\|_{\left[H^{1}\left(\Omega_{\mathrm{S}}\right)\right]^{2}} \leq\|(\mathbf{u}, \mathbf{v})\|$.

In order to prove (ii), for $\zeta \in P_{h}$, let $\bar{\zeta}:=\frac{1}{\left|\Gamma_{\mathrm{I}}\right|} \int_{\Gamma_{\mathrm{I}}} \zeta$ and $\zeta^{*}:=\zeta-\bar{\zeta}$; hence, $\int_{\Gamma_{\mathrm{I}}} \zeta^{*}=0$, both $\bar{\zeta}$ and $\zeta^{*}$ belong to $P_{h}$ and $\|\zeta\|_{L^{2}\left(\Gamma_{\mathrm{I}}\right)}^{2}=\left\|\zeta^{*}\right\|_{L^{2}\left(\Gamma_{\mathrm{I}}\right)}^{2}+\|\bar{\zeta}\|_{L^{2}\left(\Gamma_{\mathrm{I}}\right)}^{2}$.

Let $g(\mathbf{x}), \mathbf{x} \in \Gamma_{\mathrm{I}}$, be the piecewise linear function obtained by integrating $\zeta^{*}$ along $\Gamma_{\mathrm{I}}$ from a given point $\mathbf{x}_{0} \in \Gamma_{\mathrm{I}}$ to $\mathbf{x}$. Since $\int_{\Gamma_{\mathrm{I}}} \zeta^{*}=0$, then $g$ is continuous and $\|g\|_{H^{1}\left(\Gamma_{\mathrm{I}}\right)} \leq \mathbf{C}\left\|\zeta^{*}\right\|_{L^{2}\left(\Gamma_{\mathrm{I}}\right)}$.

Let $\xi \in H^{1}\left(\Omega_{\mathrm{F}}\right)$ be the solution of the Dirichlet problem

$$
\begin{aligned}
\Delta \xi & =0, & & \text { in } \Omega_{\mathrm{F}}, \\
\xi & =g, & & \text { on } \Gamma_{\mathrm{I}} .
\end{aligned}
$$

By a standard a priori estimate, $\xi \in H^{1+\varepsilon}\left(\Omega_{\mathrm{F}}\right)$ for any $\varepsilon \in\left(0, \frac{1}{2}\right)$ and $\left.\|\xi\|_{H^{1+\varepsilon}\left(\Omega_{\mathrm{F}}\right.}\right)$ $\leq\|g\|_{H^{1}\left(\Gamma_{\mathrm{I}}\right)} \leq \mathbf{C}\left\|\zeta^{*}\right\|_{L^{2}\left(\Gamma_{\mathrm{I}}\right)}$. 
Let $\xi^{\mathrm{I}} \in L_{h}\left(\Omega_{\mathrm{F}}\right)$ be the Lagrange interpolant of $\xi$; hence $\operatorname{curl} \xi^{\mathrm{I}} \cdot \boldsymbol{\nu}=\frac{\partial \xi}{\partial \boldsymbol{\tau}}=\zeta^{*}$ on $\Gamma_{\mathrm{I}}$ and $\left\|\operatorname{curl} \xi^{\mathrm{I}}\right\|_{H\left(\operatorname{div}, \Omega_{\mathrm{F}}\right)} \leq \mathbf{C}\|\xi\|_{H^{1+\varepsilon}\left(\Omega_{\mathrm{F}}\right)} \leq \mathbf{C}\left\|\zeta^{*}\right\|_{L^{2}\left(\Gamma_{\mathrm{I}}\right)}$.

On the other hand, since $\bar{\zeta} \in L^{2}\left(\Gamma_{\mathrm{I}}\right)$, we have shown in the proof of Lemma 8.1 that there exists $(\mathbf{u}, \mathbf{v}) \in \mathbf{Y}$ satisfying

$$
(\mathbf{u} \cdot \boldsymbol{\nu}-\mathbf{v} \cdot \boldsymbol{\nu})=\bar{\zeta} \quad \text { and } \quad\|(\mathbf{u}, \mathbf{v})\|_{\mathbf{Y}} \leq \mathbf{C}\|\bar{\zeta}\|_{L^{2}\left(\Gamma_{\mathrm{I}}\right)} .
$$

Now we proceed as in Theorem 5.1. Let $\mathbf{v}_{h} \in\left[L_{h}\left(\Omega_{\mathrm{S}}\right)\right]^{2}$ be the Clement's type interpolant of $\mathbf{v}$ defined therein; then $\left.\left\|\mathbf{v}_{h}\right\|_{\left[H^{1}\left(\Omega_{\mathrm{S}}\right)\right.}\right]^{2} \leq \mathbf{C}\|\mathbf{v}\|_{\left[H^{1}\left(\Omega_{\mathrm{S}}\right)\right]^{2}} \leq \mathbf{C}\|\bar{\zeta}\|_{L^{2}\left(\Gamma_{\mathrm{I}}\right)}$, $\int_{\Gamma_{\mathrm{I}}} \mathbf{v}_{h}=\int_{\Gamma_{\mathrm{I}}} \mathbf{v}$ and, hence, the following Neumann problem is compatible:

$$
\begin{aligned}
\Delta \varphi & =0, \quad \text { in } \Omega_{\mathrm{F}}, \\
\frac{\partial \varphi}{\partial \boldsymbol{\nu}} & =\mathbf{v}_{h} \cdot \boldsymbol{\nu}+\bar{\zeta}, \quad \text { on } \Gamma_{\mathrm{I}} .
\end{aligned}
$$

Its solution $\varphi \in H^{1+s}\left(\Omega_{\mathrm{F}}\right)$ with $s \in\left(\frac{1}{2}, 1\right]$ as in Theorem 4.1; thus, its gradient is smooth enough as to define its Raviart-Thomas interpolant $\mathbf{u}_{h} \in \mathbf{R}_{h}\left(\Omega_{\mathrm{F}}\right)$. This interpolant satisfies

$$
\operatorname{div} \mathbf{u}_{h}=0, \quad \int_{\ell} \mathbf{u}_{h} \cdot \boldsymbol{\nu}=\int_{\ell}\left(\mathbf{v}_{h} \cdot \boldsymbol{\nu}+\bar{\zeta}\right), \quad \forall \ell \subset \Gamma_{\mathrm{I}},
$$

and

$$
\left\|\mathbf{u}_{h}\right\|_{H\left(\operatorname{div}, \Omega_{\mathrm{F}}\right)} \leq \mathbf{C}\|\nabla \varphi\|_{\left[H^{s}\left(\Omega_{\mathrm{F}}\right)\right]^{2}} \leq \mathbf{C} \sum_{j=1}^{J}\left\|\mathbf{v}_{h} \cdot \boldsymbol{\nu}+\bar{\zeta}\right\|_{H^{1 / 2}\left(\Gamma_{j}\right)} \leq \mathbf{C}\|\bar{\zeta}\|_{L^{2}\left(\Gamma_{\mathrm{I}}\right)}
$$

(in the last inequality we have used that $\bar{\zeta}$ is constant). Now, $\left(\mathbf{u}_{h}+\operatorname{curl} \xi^{\mathrm{I}}, \mathbf{v}_{h}\right) \in$ $\mathbf{Y}_{h},\left\|\left(\mathbf{u}_{h}+\operatorname{curl} \xi^{\mathrm{I}}, \mathbf{v}_{h}\right)\right\|_{\mathbf{Y}} \leq \mathbf{C}\|\zeta\|_{L^{2}\left(\Gamma_{\mathrm{I}}\right)}$ and

$$
\int_{\Gamma_{\mathrm{I}}} \zeta\left(\left(\mathbf{u}_{h}+\operatorname{curl} \xi^{\mathrm{I}}\right) \cdot \boldsymbol{\nu}-\mathbf{v}_{h} \cdot \boldsymbol{\nu}\right)=\int_{\Gamma_{\mathrm{I}}} \zeta\left(\bar{\zeta}+\zeta^{*}\right)=\int_{\Gamma_{\mathrm{I}}} \zeta^{2}
$$

since $\left.\zeta\right|_{\ell}$ is constant for each $\ell \subset \Gamma_{\mathrm{I}}$. So, we conclude the lemma.

Now we are able to prove the convergence of the pressures at the interface. More precisely, given an eigenpair $(\lambda,(\mathbf{u}, \mathbf{v}, \mu))$ of $\mathbf{H P}$, since problems $\mathbf{H P}$ and $\mathbf{H} \mathbf{P}_{h}$ are respectively equivalent to problems $\mathbf{V P}^{*}$ and $\mathbf{V} \mathbf{P}_{h}^{*}$, by virtue of Theorems 6.5 and 6.6, there exists an eigenpair $\left(\lambda_{h},\left(\mathbf{u}_{h}, \mathbf{v}_{h}, \mu_{h}\right)\right)$ of problem $\mathbf{H P}_{h}$ such that $\left|\lambda-\lambda_{h}\right| \leq \mathbf{C} h^{2 r}$ and $\left\|(\mathbf{u}, \mathbf{v})-\left(\mathbf{u}_{h}, \mathbf{v}_{h}\right)\right\| \leq \mathbf{C}\|(\mathbf{u}, \mathbf{v})\| h^{r}$. The next theorem shows that the pressures at the interface converge with the same order as that of the displacements.

Theorem 8.1. Let $(\lambda,(\mathbf{u}, \mathbf{v}, \mu))$ and $\left(\lambda_{h},\left(\mathbf{u}_{h}, \mathbf{v}_{h}, \mu_{h}\right)\right)$ be eigenpairs of $\boldsymbol{H P}$ and $\boldsymbol{H P}_{h}$ respectively such that $\lambda_{h} \rightarrow \lambda$ as $h \rightarrow 0$ and $\left\|(\mathbf{u}, \mathbf{v})-\left(\mathbf{u}_{h}, \mathbf{v}_{h}\right)\right\| \leq \mathbf{C}\|(\mathbf{u}, \mathbf{v})\| h^{r}$. Then $\left\|\mu-\mu_{h}\right\|_{L^{2}\left(\Gamma_{\mathrm{I}}\right)} \leq \mathbf{C} h^{r}\|(\mathbf{u}, \mathbf{v})\|$, with $r$ as in Section 6 .

Proof. For all $(\phi, \psi) \in \mathbf{Y}_{h} \subset \mathbf{Y}$ we have

$$
\begin{aligned}
a^{*}\left((\mathbf{u}, \mathbf{v})-\left(\mathbf{u}_{h}, \mathbf{v}_{h}\right),(\boldsymbol{\phi}, \boldsymbol{\psi})\right)+\int_{\Gamma_{\mathrm{I}}}\left(\mu-\mu_{h}\right)(\boldsymbol{\phi} \cdot \boldsymbol{\nu}-\boldsymbol{\psi} \cdot \boldsymbol{\nu}) \\
=d\left(\lambda(\mathbf{u}, \mathbf{v})-\lambda_{h}\left(\mathbf{u}_{h}, \mathbf{v}_{h}\right),(\boldsymbol{\phi}, \boldsymbol{\psi})\right)
\end{aligned}
$$


hence for $\zeta \in P_{h}$ and $(\phi, \psi) \in \mathbf{Y}_{h}$,

$$
\begin{aligned}
\int_{\Gamma_{\mathrm{I}}}(\zeta & \left.-\mu_{h}\right)(\boldsymbol{\phi} \cdot \boldsymbol{\nu}-\boldsymbol{\psi} \cdot \boldsymbol{\nu}) \\
= & \int_{\Gamma_{\mathrm{I}}}(\zeta-\mu)(\boldsymbol{\phi} \cdot \boldsymbol{\nu}-\boldsymbol{\psi} \cdot \boldsymbol{\nu})+\int_{\Gamma_{\mathrm{I}}}\left(\mu-\mu_{h}\right)(\boldsymbol{\phi} \cdot \boldsymbol{\nu}-\boldsymbol{\psi} \cdot \boldsymbol{\nu}) \\
= & \int_{\Gamma_{\mathrm{I}}}(\zeta-\mu)(\boldsymbol{\phi} \cdot \boldsymbol{\nu}-\boldsymbol{\psi} \cdot \boldsymbol{\nu})+d\left(\lambda(\mathbf{u}, \mathbf{v})-\lambda_{h}\left(\mathbf{u}_{h}, \mathbf{v}_{h}\right),(\boldsymbol{\phi}, \boldsymbol{\psi})\right) \\
& -a^{*}\left((\mathbf{u}, \mathbf{v})-\left(\mathbf{u}_{h}, \mathbf{v}_{h}\right),(\boldsymbol{\phi}, \boldsymbol{\psi})\right),
\end{aligned}
$$

and, because of the discrete inf-sup condition in the previous lemma,

$$
\begin{aligned}
\beta \| \zeta & -\mu_{h} \|_{L^{2}\left(\Gamma_{\mathrm{I}}\right)} \leq \sup _{\substack{(\phi, \psi) \in \mathbf{Y}_{h} \\
(\phi, \psi) \neq(\mathbf{0}, \mathbf{0})}} \frac{\int_{\Gamma_{\mathrm{I}}}\left(\zeta-\mu_{h}\right)(\boldsymbol{\phi} \cdot \boldsymbol{\nu}-\boldsymbol{\psi} \cdot \boldsymbol{\nu})}{\|(\boldsymbol{\phi}, \boldsymbol{\psi})\|_{\mathbf{Y}}} \\
& \leq\|\zeta-\mu\|_{L^{2}\left(\Gamma_{\mathrm{I}}\right)}+\mathbf{C}\left[\left|\lambda(\mathbf{u}, \mathbf{v})-\lambda_{h}\left(\mathbf{u}_{h}, \mathbf{v}_{h}\right)\right|+\left\|(\mathbf{u}, \mathbf{v})-\left(\mathbf{u}_{h}, \mathbf{v}_{h}\right)\right\|\right] .
\end{aligned}
$$

Therefore, by using the triangle inequality,

$$
\begin{aligned}
\| \mu- & \mu_{h} \|_{L^{2}\left(\Gamma_{\mathrm{I}}\right)} \\
& \leq \mathbf{C}\left[\inf _{\zeta \in P_{h}}\|\zeta-\mu\|_{L^{2}\left(\Gamma_{\mathrm{I}}\right)}+\left|\lambda(\mathbf{u}, \mathbf{v})-\lambda_{h}\left(\mathbf{u}_{h}, \mathbf{v}_{h}\right)\right|+\left\|(\mathbf{u}, \mathbf{v})-\left(\mathbf{u}_{h}, \mathbf{v}_{h}\right)\right\|\right] .
\end{aligned}
$$

By using Theorem 6.6 and the assumed bound on $\left\|(\mathbf{u}, \mathbf{v})-\left(\mathbf{u}_{h}, \mathbf{v}_{h}\right)\right\|$, the two last terms in the right hand side can be bounded by $\mathbf{C} h^{r}\|(\mathbf{u}, \mathbf{v})\|$. On the other hand, by using Theorem 4.3 , we know that $p \in H^{1+s}\left(\Omega_{\mathrm{F}}\right)$ for $s>1 / 2$ with $\|p\|_{H^{1+s}\left(\Omega_{\mathrm{F}}\right)} \leq$ $|(\mathbf{u}, \mathbf{v})|$ and, hence, $\mu=\left.p\right|_{\Gamma_{\mathrm{I}}} \in H^{1}\left(\Gamma_{\mathrm{I}}\right)$. Therefore,

$$
\inf _{\zeta \in P_{h}}\|\zeta-\mu\|_{L^{2}\left(\Gamma_{\mathrm{I}}\right)} \leq \mathbf{C} h\|\mu\|_{H^{1}\left(\Gamma_{\mathrm{I}}\right)}=\mathbf{C} h\|p\|_{H^{1}\left(\Gamma_{\mathrm{I}}\right)} \leq \mathbf{C} h|(\mathbf{u}, \mathbf{v})| .
$$

Consequently, $\left\|\mu-\mu_{h}\right\|_{L^{2}\left(\Gamma_{\mathrm{I}}\right)} \leq \mathbf{C} h^{r}\|(\mathbf{u}, \mathbf{v})\|$.

The computed approximation of the pressure at the interface can be used to recover the pressure into the fluid by solving a Dirichlet problem by means of piecewise linear continuous finite elements. However $\mu_{h} \notin H^{1 / 2}\left(\Gamma_{\mathrm{I}}\right)$; moreover, $\mu_{h}$ is discontinuous at the vertices on $\Gamma_{\mathrm{I}}$ and these nodal values are needed to solve the discrete problem. Therefore, $\mu_{h}$ should be post-processed.

For each vertex $B \in \Gamma_{\mathrm{I}}$, let $\ell_{B}^{-}$and $\ell_{B}^{+}$be the two edges on $\Gamma_{\mathrm{I}}$ sharing $B$. We define $\mu_{h}^{*}$ as the piecewise linear continuous function on $\Gamma_{\mathrm{I}}$ with average nodal values

$$
\mu_{h}^{*}(B):=\frac{1}{2}\left(\left.\mu_{h}\right|_{\ell_{B}^{-}}+\left.\mu_{h}\right|_{\ell_{B}^{+}}\right) .
$$

Let $\Lambda_{h}: L^{2}\left(\Gamma_{\mathrm{I}}\right) \longrightarrow L_{h}\left(\Gamma_{\mathrm{I}}\right):=\left\{\zeta \in H^{1}\left(\Gamma_{\mathrm{I}}\right):\left.\zeta\right|_{\ell} \in \mathcal{P}_{1}(\ell), \forall \ell \subset \Gamma_{\mathrm{I}}\right\}$ be the Clement's type interpolation defined by the nodal values

$$
\Lambda_{h} \zeta(B):=\frac{1}{2}\left(\frac{1}{\left|\ell_{B}^{-}\right|} \int_{\ell_{B}^{-}} \zeta+\frac{1}{\left|\ell_{B}^{+}\right|} \int_{\ell_{B}^{+}} \zeta\right) ;
$$


then $\mu_{h}^{*}=\Lambda_{h} \mu_{h}$ and, by using the techniques in [6], it is straightforward to show that

$$
\begin{aligned}
\left\|\Lambda_{h} \zeta\right\|_{L^{2}\left(\Gamma_{\mathrm{I}}\right)} & \leq\|\zeta\|_{L^{2}\left(\Gamma_{\mathrm{I}}\right)}, \quad \forall \zeta \in L^{2}\left(\Gamma_{\mathrm{I}}\right), \\
\left\|\zeta-\Lambda_{h} \zeta\right\|_{L^{2}\left(\Gamma_{\mathrm{I}}\right)} & \leq \mathbf{C} h\|\zeta\|_{H^{1}\left(\Gamma_{\mathrm{I}}\right)}, \quad \forall \zeta \in H^{1}\left(\Gamma_{\mathrm{I}}\right)
\end{aligned}
$$

By using these estimates, the fact that $\mu=\left.p\right|_{\Gamma_{\mathrm{I}}}$, a standard trace theorem, Theorem 8.1 and Theorem 4.3, we have:

$$
\begin{aligned}
\left\|\mu-\mu_{h}^{*}\right\|_{L^{2}\left(\Gamma_{\mathrm{I}}\right)} & \leq\left\|\mu-\Lambda_{h} \mu\right\|_{L^{2}\left(\Gamma_{\mathrm{I}}\right)}+\left\|\Lambda_{h}\left(\mu-\mu_{h}\right)\right\|_{L^{2}\left(\Gamma_{\mathrm{I}}\right)} \\
& \leq \mathbf{C} h\|\mu\|_{H^{1}\left(\Gamma_{\mathrm{I}}\right)}+\left\|\left(\mu-\mu_{h}\right)\right\|_{L^{2}\left(\Gamma_{\mathrm{I}}\right)} \\
& \leq \mathbf{C} h\|p\|_{H^{3 / 2}\left(\Omega_{\mathrm{F}}\right)}+\mathbf{C} h^{r}\|(\mathbf{u}, \mathbf{v})\| \\
& \leq \mathbf{C} h^{r}\|(\mathbf{u}, \mathbf{v})\|
\end{aligned}
$$

Therefore, $\mu_{h}^{*}$ gives an approximation of $\left.p\right|_{\Gamma_{\mathrm{I}}}$ of the same order as $\mu_{h}$ in $L^{2}\left(\Gamma_{\mathrm{I}}\right)$.

Now, we can approximate the pressure into the fluid by means of the solution $\tilde{p}_{h} \in L_{h}\left(\Omega_{\mathrm{F}}\right)$ of the following discrete Dirichlet problem:

$$
\begin{aligned}
\int_{\Omega_{\mathrm{F}}} \nabla \tilde{p}_{h} \cdot \nabla \zeta & =0, \quad \forall \zeta \in L_{h}^{0}\left(\Omega_{\mathrm{F}}\right), \\
\tilde{p}_{h} & =\mu_{h}^{*}, \quad \text { on } \Gamma_{\mathrm{I}},
\end{aligned}
$$

where $L_{h}^{0}\left(\Omega_{\mathrm{F}}\right):=\left\{\zeta \in L_{h}\left(\Omega_{\mathrm{F}}\right): \zeta=0\right.$ on $\left.\Gamma_{\mathrm{I}}\right\}$.

We have the following error estimates:

Theorem 8.2. If the family of meshes $\left\{\mathcal{T}_{h}\right\}$ is quasiuniform, then

$$
\begin{array}{r}
\left\|p-\tilde{p}_{h}\right\|_{L^{2}\left(\Omega_{\mathrm{F}}\right)} \leq \mathbf{C} h^{r}\|(\mathbf{u}, \mathbf{v})\|, \\
\left\|\nabla\left(p-\tilde{p}_{h}\right)\right\|_{L^{2}\left(\Omega_{\mathrm{F}}\right)} \leq \mathbf{C} h^{r-1 / 2}\|(\mathbf{u}, \mathbf{v})\|,
\end{array}
$$

where $r:=\min \{s, t\}$ with $s$ and $t$ as in Theorem 4.1.

Proof. We prove first the second inequality. Let $p^{\mathrm{I}}$ be the Lagrange interpolant of $p$. We extend $\mu_{h}^{*}$ to the interior of $\Omega_{\mathrm{F}}$ as a piecewise linear continuous function by defining $\mu_{h}^{*}(B):=p(B)$ for all the interior nodes $B \in \Omega_{\mathrm{F}}$ of the triangulation. Standard arguments show that $\left\|\nabla\left(p-\tilde{p}_{h}\right)\right\|_{\left[L^{2}\left(\Omega_{\mathrm{F}}\right)\right]^{2}} \leq\left\|\nabla\left(p-\mu_{h}^{*}\right)\right\|_{\left[L^{2}\left(\Omega_{\mathrm{F}}\right)\right]^{2}}$ and hence

$$
\left.\left\|\nabla\left(p-\tilde{p}_{h}\right)\right\|_{\left[L^{2}\left(\Omega_{\mathrm{F}}\right)\right]^{2}} \leq\left\|\nabla\left(p-p^{\mathrm{I}}\right)\right\|_{\left[L^{2}\left(\Omega_{\mathrm{F}}\right)\right.}\right]^{2}+\left\|\nabla\left(p^{\mathrm{I}}-\mu_{h}^{*}\right)\right\|_{\left[L^{2}\left(\Omega_{\mathrm{F}}\right)\right]^{2}} .
$$

Since

$$
\left.\left\|\nabla\left(p-p^{\mathrm{I}}\right)\right\|_{\left[L^{2}\left(\Omega_{\mathrm{F}}\right)\right.}\right]^{2} \leq \mathbf{C} h^{s}\|p\|_{H^{1+s}\left(\Omega_{\mathrm{F}}\right)} \leq \mathbf{C} h^{s}\|(\mathbf{u}, \mathbf{v})\|
$$

and $s>\frac{1}{2} \geq r-\frac{1}{2}$, then we only need to estimate the second term in the right hand side of (8.4). 
For any $B \in \Gamma_{\mathrm{I}}$, node of $\mathcal{T}_{h}$, let $\varphi_{B}$ be the basis function of $L_{h}\left(\Omega_{\mathrm{F}}\right)$ associated to $B$. Since $p^{\mathrm{I}}$ and $\mu_{h}^{*}$ coincide at the interior nodes we have $p^{\mathrm{I}}-\mu_{h}^{*}=$ $\sum_{B \in \Gamma_{\mathrm{I}}}\left[p^{\mathrm{I}}(B)-\mu_{h}^{*}(B)\right] \varphi_{B}$ and then

$$
\begin{aligned}
\left.\left\|\nabla\left(p^{\mathrm{I}}-\mu_{h}^{*}\right)\right\|_{\left[L^{2}\left(\Omega_{\mathrm{F}}\right)\right.}^{2}\right]^{2} & \left.\leq \sum_{B \in \Gamma_{\mathrm{I}}}\left|p^{\mathrm{I}}(B)-\mu_{h}^{*}(B)\right|^{2}\left\|\nabla \varphi_{B}\right\|_{\left[L^{2}\left(\Omega_{\mathrm{F}}\right)\right.}^{2}\right]^{2} \\
& \leq \mathbf{C} \sum_{\ell \subset \Gamma_{\mathrm{I}}}\left\|p^{\mathrm{I}}-\mu_{h}^{*}\right\|_{L^{\infty}(\ell)}^{2},
\end{aligned}
$$

where in the last inequality we have used that $\left.\left\|\nabla \varphi_{B}\right\|_{\left[L^{2}\left(\Omega_{\mathrm{F}}\right)\right.}\right]^{2}$ is bounded independently of $h$. By using an inverse inequality,

$$
\left\|p^{\mathrm{I}}-\mu_{h}^{*}\right\|_{L^{\infty}(\ell)} \leq \mathbf{C}|\ell|^{-1 / 2}\left\|p^{\mathrm{I}}-\mu_{h}^{*}\right\|_{L^{2}(\ell)}
$$

and hence

$$
\left.\left\|\nabla\left(p^{\mathrm{I}}-\mu_{h}^{*}\right)\right\|_{\left[L^{2}\left(\Omega_{\mathrm{F}}\right)\right.}^{2}\right]^{2} \leq \mathbf{C} \sum_{\ell \subset \Gamma_{\mathrm{I}}} \frac{1}{|\ell|}\left\|p^{\mathrm{I}}-\mu_{h}^{*}\right\|_{L^{2}(\ell)}^{2} .
$$

Since the mesh is assumed to be quasiuniform, $|\ell| \geq \mathbf{C} h$ for any edge $\ell$ of the triangulation and hence

$$
\begin{aligned}
\left\|\nabla\left(p^{\mathrm{I}}-\mu_{h}^{*}\right)\right\|_{L^{2}\left(\Omega_{\mathrm{F}}\right)} & \leq \mathbf{C} h^{-1 / 2}\left\|p^{\mathrm{I}}-\mu_{h}^{*}\right\|_{L^{2}\left(\Gamma_{\mathrm{I}}\right)} \\
& \leq \mathbf{C} h^{-1 / 2}\left[\left\|p^{\mathrm{I}}-p\right\|_{L^{2}\left(\Gamma_{\mathrm{I}}\right)}+\left\|p-\mu_{h}^{*}\right\|_{L^{2}\left(\Gamma_{\mathrm{I}}\right)}\right] \\
& \leq \mathbf{C} h^{-1 / 2}\left[h\|p\|_{H^{1}\left(\Gamma_{\mathrm{I}}\right)}+h^{r}\|(\mathbf{u}, \mathbf{v})\|\right] \\
& \leq \mathbf{C} h^{r-1 / 2}\|(\mathbf{u}, \mathbf{v})\|,
\end{aligned}
$$

where we have used standard error estimates for $\left.\left(p-p^{\mathrm{I}}\right)\right|_{\Gamma_{\mathrm{I}}},(8.3)$ and the a priori estimate for $p$. Therefore, by using (8.4), (8.5) and (8.6), since $r-1 / 2<s$, we prove the second estimate of the theorem.

Finally, a standard duality argument shows that

$$
\left\|p-\tilde{p}_{h}\right\|_{L^{2}\left(\Omega_{\mathrm{F}}\right)} \leq \mathbf{C} h^{s}\left\|\nabla\left(p-\tilde{p}_{h}\right)\right\|_{L^{2}\left(\Omega_{\mathrm{F}}\right)}+\mathbf{C} h^{r}\left\|p-\mu_{h}^{*}\right\|_{L^{2}\left(\Gamma_{\mathrm{I}}\right)} \leq \mathbf{C} h^{r}\|(\mathbf{u}, \mathbf{v})\| .
$$

Let us remark that whenever the singularities of the solution in the structure are not too strong, $r>1 / 2$ (see [10]), and hence this theorem shows that $\nabla \tilde{p}_{h}$ also converges to $\nabla p$.

\section{Conclusions}

We have introduced a finite element method able to solve internal fluid-structure vibration problems for compressible as well as for incompressible fluids. In both cases we have proved convergence and given optimal error estimates for eigenmodes and eigenfrequencies. We have also proved that this method does not present spurious modes.

In the case of compressible fluids, the approach in this paper can be seen as a theoretical alternative to that in $[1,3]$ allowing to obtain error estimates not depending on the acoustic speed. 
An asymptotic analysis shows that the eigenfrequencies for a problem with an incompressible fluid are limits of those for a compresssible one when the acoustic speed goes to infinity. This, being true in the continuous as well as in the discrete problem, allows to deal with a nearly incompressible fluid by approximating it with a perfectly incompressible one.

In the incompressible case, a discretization based on the stream function of the fluid displacements is introduced to save computational effort. A hybrid implementation of this method and a convenient computation of the pressure in the fluid have been also analyzed. Numerical results for this problem are reported in [2].

\section{ACKNowledgement}

The authors want to express their gratitude to the referee for very helpful suggestions.

\section{REFERENCES}

1. A. Bermúdez, R. Durán, M.A. Muschietti, R. Rodríguez and J. Solomin, Finite element vibration analysis of fluid-solid systems without spurious modes. SIAM J. Numér. Anal., 32 (1995) 1280-1295. MR 96e:73072

2. A. Bermúdez, R. Durán and R. Rodríguez, Finite element solution of incompressible fluidstructure vibration problems. Int. J. Numer. Methods Eng. 40 (1997) 1435-1448. CMP 97:10

3. A. Bermúdez and R. Rodríguez, Finite element computation of the vibration modes of a fluidsolid system, Comp. Methods in Appl. Mech. and Eng. 119 (1994) 355-370. MR 95j:73064

4. J. Boujot, Mathematical formulation of fluid-structure interaction problems, RAIRO Modél. Math. Anal. Numér. 21 (1987) 239-260. MR 88j:73032

5. F. Brezzi, M. Fortin, Mixed and Hybrid Finite Element Methods, Springer-Verlag, New York, 1991. MR 92d:65187

6. P. Clement, Approximation by finite element functions using local regularization, RAIRO Anal. Num. 9 (1975) 77-84. MR 53:4569

7. J. Descloux, N. Nassif and J. Rappaz, On spectral approximation. Part 1: The problem of convergence. Part 2: Error estimates for the Galerkin methods, RAIRO Anal. Numér. 12 (1978) 97-119. MR 58:3404a; MR 58:3404b

8. T. Geveci, B. D. Reddy and H. T. Pearce, On the approximation of the spectrum of the Stokes operator, RAIRO Modél. Math. Anal. Numér. 23 (1989) 129-136. MR 90i:65186

9. V. Girault and P.A. Raviart, Finite element methods for Navier-Stokes equations, SpringerVerlag, Berlin, Heidelberg, New York, Tokyo, 1986. MR 88b:65129

10. P. Grisvard, Elliptic problems for non-smooth domains, Pitman, Boston, 1985. MR 86m:35044

11. M. Hamdi, Y. Ouset and G. Verchery, A displacement method for the analysis of vibrations of coupled fluid-structure systems, Int. J. Numer. Methods Eng. 13 (1978) 139-150.

12. H.J-P. Morand and R. Ohayon, Interactions fluides-structures, Recherches en Mathématiques Appliquées 23, Masson, Paris, 1992. MR 93m:73031

13. R. Ohayon and E. Sánchez-Palencia, On the vibration problem for an elastic body surrounded by a slightly compressible fluid. RAIRO Modél. Math. Anal. Numér. 17 (1983) 311-326. MR 84f: 73030

14. R. Rodríguez and J. Solomin, The order of convergence of eigenfrequencies in finite element approximations of fluid-structure interaction problems. Math. Comp. 65 (1996) 1463-1475. MR 97a:65090

15. P. A. Raviart and J. M. Thomas, A mixed finite element method for second order elliptic problems, Mathematical Aspects of Finite Element Methods. Lecture Notes in Mathematics 606, Springer Verlag, Berlin, Heidelberg, New York, 1977, 292-315. MR 58:3547

16. J. Sánchez-Hubert and E. Sánchez-Palencia, Vibration and Coupling of Continuous Systems. Asymptotic Methods, Springer-Verlag, Berlin, 1989. MR 91c:00018 
17. L. R. Scott and S. Zhang, Finite element interpolation of nonsmooth functions satisfying boundary conditions, Math. Comp. 54 (1990) 483-493. MR 90j:65021

Departamento de Matemática Aplicada, Universidade de Santiago de Compostela, 15706 Santiago de Compostela, Spain

E-mail address: bermudez@zmat.usc.es

Departamento de Matemática, Facultad de Ciencias Exactas y Naturales, UniversiDad de Buenos Aires, 1428 - Buenos Aires, Argentina

E-mail address: rduran@mate.dm.uba.ar

Departamento de Ingeniería Matemática, Universidad de Concepción, Casilla 4009, CONCEPCión, Chile

E-mail address: rodolfo@gauss.cfm.udec.cl 